\title{
OPTIMAL POLICIES FOR A DETERMINISTIC CONTINUOUS-TIME INVENTORY MODEL WITH SEVERAL SUPPLIERS
}

\author{
LAKDERE Benkherouf ${ }^{1, *}$ And Brian H. Gilding ${ }^{2}$
}

\begin{abstract}
This paper is concerned with determining the optimal inventory policy for an infinitehorizon deterministic continuous-time continuous-state inventory model, where, in the absence of intervention, changes in inventory level are governed by a differential evolution equation. The decision maker has the option of ordering from several suppliers, each of which entails differing ordering and purchasing costs. The objective is to select the supplier and the size of the order that minimizes the discounted cost over an infinite planning horizon. The optimal policy is formulated as the solution of a quasi-variational inequality. It is shown that there are three possibilities regarding its solvability: it has a unique solution that corresponds to an $(s, S)$ policy; it does not admit a solution corresponding to an $(s, S)$ policy but does have a unique solution that corresponds to a generalized $(s, S)$ policy; or, it does not admit a solution corresponding to an $(s, S)$ policy or a generalized $(s, S)$ policy. A necessary and sufficient condition for each possibility is obtained. Examples illustrate their occurrence.
\end{abstract}

Mathematics Subject Classification. 90B05.

Received November 2, 2019. Accepted April 4, 2020.

\section{INTRODUCTION}

Inventory control is concerned with the efficient management of stock of diverse items that are used in a production process or the provision of a service. Such items may include raw materials, components for work in progress, finished products, and consumables. The capital tied up with stocking such items is large enough to call for the application of quantitative methods of management. Holding costs influence the balance between variation in supply of the stock and demand.

A policy in inventory control that has proven to be practical and effective, and has stood the test of time, is the $(s, S)$ policy. This policy sets two levels of stock denoted by $s$ and $S>s$. The policy recommends ordering to bring the level up to $S$ whenever it drops to $s$, and not replenishing when it exceeds $s$. Such a policy was introduced in [1]. A policy of this type was shown to be optimal in each period of a multi-period dynamic inventory problem with stochastic demand if the holding and shortage costs are linear functions of the level of stock, and, in a single period problem if the expected cost function is a concave function of the level of stock, in the well-known paper [21]. Assumptions in this paper regarding the differentiability of certain cost

Keywords. Optimal inventory policy, quasi-variational inequality, $(s, S)$ policy, generalized $(s, S)$ policy.

1 Department of Statistics and Operations Research, College of Science, Kuwait University, P.O. Box 5969, Safat 13060, Kuwait.

2 Department of Mathematics, College of Science, Kuwait University, P.O. Box 5969, Safat 13060, Kuwait.

* Corresponding author: lakdere. benkherouf @ku .edu.kw 
functions were subsequently relaxed in [28]. An $(s, S)$ policy was further shown to be optimal in an infinitehorizon dynamic inventory model in [13]. All of the aforementioned results were generalized and shown to be valid under alternative conditions in [25]. For a discrete-time stationary inventory model, an $(s, S)$ policy was shown to be optimal using a quasi-variational inequality (QVI) in [3]. Similar results confirming the optimality of an $(s, S)$ policy using a QVI were obtained in $[4,7]$.

A variation on the $(s, S)$ inventory policy is the $(s, S, T)$ periodic review inventory policy, which was introduced in [11]. Incorporating a cost as the consequence of a review, the policy is to bring the level of stock up to $S>s$ when it is $s$ or less, and take no action otherwise, with period $T$. For practical applications, particularly those involving a stochastic demand that is expensive to monitor, such a policy has operational benefits. Policies of this type are studied in [14] and references cited therein.

Another variation on the $(s, S)$ inventory policy is what has become known in the inventory control literature as the generalized $(s, S)$ policy. It involves several suppliers, and stock levels $s_{N}<s_{N-1}<\cdots<s_{1}<S_{1}<$ $S_{2}<\cdots<S_{N}$ for some natural number $N$. If the level of stock is greater than $s_{1}$ one does not replenish it, if it is between $s_{2}$ and $s_{1}$ one orders from supplier 1 to complete the stock to level $S_{1}$, and so on, down to a level of stock between $s_{N}$ and $s_{N-1}$ where one orders from supplier $N-1$ to bring the stock up to level $S_{N-1}$. If the stock level is less than $s_{N}$ one orders from supplier $N$ to complete the stock to level $S_{N}$. The policy may intentionally exclude further available suppliers. Introductions to generalized $(s, S)$ policies may be found in $[5,19]$.

Although a generalized $(s, S)$ policy seems to be very practical in real life, there are few research articles on the topic. A discrete-time stochastic inventory model with an ordering-cost function that is concave is considered in $[17,18]$. A dynamic model with two suppliers, one with a high purchasing cost and no setup cost, and the other with a low purchasing cost and a fixed ordering cost, is examined in [9]. With a finite and with an infinite horizon, the existence of a generalized $(s, S)$ policy is shown under the assumption that the probability density function of the demand is log-concave. This assumption is met by many probability distributions, including the normal and gamma distributions. More recently, the optimal policy for the model treated in [17] with a general demand distribution has been shown to be a generalized $(s, S)$ policy for all but a finite interval of inventory levels, in [2].

In the present paper, an inventory manager is faced with the problem of determining an optimal replenishment policy for a continuous-time continuous-state deterministic inventory model with a single item, where, in the absence of intervention, the changes in the inventory level are modelled by a differential equation, and where the control policies are of an impulse control type. Replenishment is offered by a number of suppliers, each of which charges differently. The objective is to find an admissible control that minimizes the overall inventory costs over an infinite planning horizon. For a single supplier model the optimal policy is of $(s, S)$ type. However, when several suppliers are available, the problem is far from straightforward. As it turns out, there are three possibilities. Either there is a unique optimal policy that is an $(s, S)$ policy, there is a unique optimal policy that is a generalized $(s, S)$ policy but not an $(s, S)$ policy, or, an optimal policy is neither of these. The technical machinery employed to solve the problem is based on QVI techniques. A model related to that studied in the present paper has been investigated in [23]. For further information on QVIs, the reader is referred to $[6,8]$.

Our results differ from those in $[12,15,16,26,27]$. These papers deal with the long-term average cost criterion for continuous-time models, and indicate that under the respective circumstances an $(s, S)$ policy is optimal. This is not the case, in general, for the infinite-horizon discounted model considered in the present paper.

The next section contains the formal statement of the inventory control problem as well as the QVI formulation. The derivation of the optimality of the $(s, S)$ policy for a single supplier is reviewed in Section 3 as a prelude to the main contribution of the paper. The analysis of the problem with several suppliers is subsequently to be found in Section 4. Examples 4.22-4.24 provide concrete illustrations of the three possible outcomes alluded to above. A conclusion and some general remarks are provided in Section 5. 


\section{Problem statement}

Consider a stock of a single item with $J$ possible suppliers. The level of stock at time $t$ is given by $x(t)$. A level $x \geq 0$ corresponds to the number of items held. A level $x<0$ indicates a shortage of $-x$ items. In the absence of intervention, changes in $x$ are governed by the evolution equation

$$
\dot{x}(t)=-G(x(t)),
$$

where $G$ is a positive continuous function defined on $\mathbb{R}$. From a modelling point of view, the latter accounts for a stock-dependent demand rate and the deterioration of items. The forms of $G$ that are commonly used have been reviewed in $[10,24]$. A generic expression that amalgamates these forms is

$$
G(x)= \begin{cases}g_{0} & \text { for } \quad x<0 \\ g_{0}+g_{1} x+g_{\beta} x^{\beta} & \text { for } \quad x \geq 0,\end{cases}
$$

where $g_{0}>0, g_{1} \geq 0, g_{\beta} \geq 0$, and $0<\beta<1$ are prescribed constants.

Placing an order with supplier $j \in \mathcal{J}$, where

$$
\mathcal{J}=\{1,2, \ldots, J\},
$$

entails a fixed cost $k_{j}$ and a cost $c_{j}$ per unit item. Given a choice between two suppliers $j$ and $\ell$, for which $k_{j}<k_{\ell}$ and $c_{j} \leq c_{\ell}$, or for which $k_{j} \leq k_{\ell}$ and $c_{j}<c_{\ell}$, one would obviously opt for supplier $j$. Also, as far as minimizing costs is involved, given a choice between two suppliers $j$ and $\ell$ with $k_{j}=k_{\ell}$ and $c_{j}=c_{\ell}$, it is immaterial which of them one chooses. So, with no loss of generality, it will be supposed that

$$
k_{1}>k_{2}>\cdots>k_{J}>0
$$

and

$$
c_{1}<c_{2}<\cdots<c_{J} .
$$

Under this assumption using a supplier with a higher set-up cost involves a lesser cost per item. For any supplier, this may well be the case with regard to a larger competitor whose warehouse is located further away from the customer. Whereas purchasing from the competitor requires a higher cost for transportation, say, which is then transferred into the set-up cost, the price charged per unit by the competitor could be lower.

The holding cost is given by a continuous nonnegative function $f$ defined on $\mathbb{R}$. The classical expression is

$$
f(x)=\left\{\begin{array}{lll}
-p x & \text { for } \quad x<0 \\
q x & \text { for } \quad x \geq 0
\end{array}\right.
$$

where $p>0$ and $q>0$ are constants. This is a linear function of the inventory level for items in stock, and for a shortage of items.

Variability of money-value in time is considered by the exponential discount of costs at a constant rate

$$
\alpha>0 \text {. }
$$

This method of discounting costs was proposed in [20], is an integral component of the continuous-time inventory models in $[4,5,7,8,22,23]$, and is possibly the most popular method of discounting applied in economics.

An admissible replenishment strategy consists of a sequence

$$
V_{n}=\left\{\left(t_{i}, \xi_{i}, j_{i}\right): i=1, \ldots, n\right\},
$$

where $t_{i} \geq 0$ represents the time of the $i$ th control (intervention), $\xi_{i}>0$ is the quantity ordered at that time, and $j_{i} \in \mathcal{J}$ labels the supplier. One may note that should one wish to order a total of $\Xi$ items from a selection 
of suppliers $\mathcal{I} \subseteq \mathcal{J}$ at the same time, then the minimum of the total cost $\sum_{j \in \mathcal{I}}\left(k_{j}+c_{j} \xi_{j}\right)$ subject to the constraints $\xi_{j} \geq 0$ for every $j \in \mathcal{I}$ and $\sum_{j \in \mathcal{I}} \xi_{j}=\Xi>0$ is necessarily given by a combination in which $\xi_{j}=\Xi$ for some $j \in \mathcal{I}$, and $\xi_{\ell}=0$ for every other $\ell \in \mathcal{I}$ [5]. Thus, at any time $t_{i}$, an order will be placed with a single supplier.

In this setting, the problem of finding an optimal impulse control policy reduces to that of determining a sequence

$$
V=\lim _{n \rightarrow \infty} V_{n}
$$

that solves

$$
u(x(0))=\min _{V}\left\{\int_{0}^{\infty} f(x(t)) \mathrm{e}^{-\alpha t} \mathrm{~d} t+\sum_{i=1}^{\infty}\left(k_{j_{i}}+c_{j_{i}} \xi_{i}\right) \mathrm{e}^{-\alpha t_{i}}\right\} .
$$

Note that if $x$ satisfies equation (2.1) then

$$
\tilde{x}=\int_{0}^{x} \frac{\mathrm{d} \eta}{G(\eta)}
$$

satisfies the same equation with $G$ replaced by the constant function 1. Concurrently, if $u$ satisfies (2.7) then $\tilde{u}(\tilde{x}(0))=u(x(0))$ satisfies $(2.7)$ with $f(x(t))$ replaced by $\tilde{f}(\tilde{x}(t))$. Conversely, by the reverse transformation, any solution of (2.7) in which $x$ is governed by (2.1) with $G \equiv 1$ gives rise to a solution satisfying the original equation (2.1) if

$$
\int_{0}^{x} \frac{\mathrm{d} \eta}{G(\eta)} \rightarrow \pm \infty \quad \text { as } \quad x \rightarrow \pm \infty
$$

This condition is fulfilled by (2.2). Hence, with nominal loss of generality, we shall take $G \equiv 1$.

With the aforementioned simplification, it will be shown that the optimal impulse control policy of $(2.7)$ corresponds to a solution of a QVI. If no order is made within a time interval from $t$ to $t+\tau$ where $\tau>0$, then (2.1) with $G \equiv 1$ and (2.7) lead to

$$
\begin{aligned}
u(x(t)) & \leq u(x(t+\tau)) \mathrm{e}^{-\alpha \tau}+\int_{t}^{t+\tau} f(x(\rho)) \mathrm{e}^{-\alpha(\rho-t)} \mathrm{d} \rho \\
& =u(x(t)-\tau) \mathrm{e}^{-\alpha \tau}+\int_{0}^{\tau} f(x(t)-v) \mathrm{e}^{-\alpha v} \mathrm{~d} v .
\end{aligned}
$$

Hence, dropping the dependence of $x$ on $t$,

$$
u(x)-u(x-\tau)+\alpha \tau u(x-\tau) \leq \alpha u(x-\tau) \int_{0}^{\tau}\left(1-\mathrm{e}^{-\alpha v}\right) \mathrm{d} v+\int_{0}^{\tau} f(x-v) \mathrm{e}^{-\alpha v} \mathrm{~d} v .
$$

Dividing by $\tau$, and then letting $\tau \rightarrow 0$, yields $(A u)(x) \leq f(x)$, where

$$
(A u)(x)=u^{\prime}(x)+\alpha u(x) .
$$

On the other hand, if an order is made with supplier $j$ at time $t$, then the quantity ordered should minimize $k_{j}+u(x(t)+\xi)+c_{j} \xi$ with respect to $\xi \geq 0$. In other words, one should have $u(x(t))=\left(M_{j} u\right)(x(t))$, where

$$
\left(M_{j} u\right)(x)=k_{j}+\min \left\{u(x+\xi)+c_{j} \xi: \xi \geq 0\right\} .
$$

We deduce that the optimal impulse control policy corresponds to the solution of the QVI

$$
\left\{\begin{array}{l}
A u \leq f \\
u \leq M u \\
(A u-f)(u-M u)=0,
\end{array}\right.
$$

in which $A$ is given by (2.8), and

$$
(M u)(x)=\min \left\{\left(M_{j} u\right)(x): j \in \mathcal{J}\right\} .
$$

To show the existence of a solution of the QVI (2.9) it will be helpful to first examine the case where $J=1$. 


\section{The $(s, S)$ POLICY FOR A SINGLE SUPPliER}

When there is a single supplier, we may identify $M$ with $M_{1}$, and drop the subscript from $k_{1}$ and $c_{1}$. Subsequently, it can be verified that if $u$ satisfies the QVI (2.9), then the function $\tilde{u}$ given by $\tilde{u}(x)=u(x)+$ $c x-c / \alpha$ satisfies (2.9) with $f$ replaced by $\tilde{f}$, where $\tilde{f}(x)=f(x)+\alpha c x$, and $c$ replaced by 0 , and vice versa. Hence, with no loss of generality, we may suppose that $c=0$.

In the expectation that the optimal inventory control policy will involve levels of stock $x$ defining a stopping interval in which $u=M u$, and levels of stock $x$ not necessitating replenishment defining an interval in which $u<M u$ and $A u=f$, separated by some stock level $s$, we search for a solution of the QVI based on the following premise.

Ansatz 3.1. The solution of (2.9) is a differentiable real function $u$ such that $u=M u$ on $(-\infty, s]$, and, $A u=f$ and $u<M u$ in $(s, \infty)$, for some number $s$.

Lemma 3.2. Under Ansatz 3.1, a solution of the QVI is given by

$$
u(x)=\left\{\begin{array}{lll}
y(s) & \text { for } & x<s \\
y(x) & \text { for } & x \geq s,
\end{array}\right.
$$

where $y$ is a solution of the differential equation

$$
y^{\prime}+\alpha y=f \quad \text { in } \quad \mathbb{R}
$$

satisfying

$$
y^{\prime}(s)=y^{\prime}(S)=0
$$

and

$$
y(s)=y(S)+k
$$

for some number $S>s$.

Proof. Suppose that $u$ is a solution of the QVI that satisfies the ansatz. Let $x<s$. Then, since $(M u)(x)$ is well defined, $u$ must have a least absolute minimum in $[x, \infty)$, at $S$ say. Because this implies that $u(x)=(M u)(x)=$ $k+u(S)$, necessarily $S>x$. In turn this means that $u$ has a least absolute minimum in $[\eta, \infty)$ at $S$ for all $x \leq \eta \leq S$. Whence, $u(\eta)=(M u)(\eta)=k+u(S)$ for all $x \leq \eta \leq \min \{s, S\}$, and, $u(\min \{s, S\})=k+u(S)$. It follows that $S>s$, that $S$ is the least absolute minimum of $u$ on $[s, \infty)$, that $u(s)=k+u(S)$, and, in view of the arbitrariness of $x<s$, that $u$ is constant on $(-\infty, s]$. Given the differentiability of $u$, the minimality of $S$ requires $u^{\prime}(S)=0$, and the constance of $u$ on $(-\infty, s]$ requires $u^{\prime}(s)=0$. Moreover, the condition $A u=f$ in $(s, \infty)$ says that $u$ must be a solution of (3.2) there. However, under the assumption that $f$ is a continuous function on $\mathbb{R}$, any solution of the ordinary differential equation $(3.2)$ in $(s, \infty)$ can be extended onto $\mathbb{R}$. Thus $u$ must be as in (3.1) with $y$ as stated.

The following hypothesis is sufficient for the existence of a function $y$ as indicated in Lemma 3.2 .

Hypothesis 3.3. The function $f$ is continuous on $\mathbb{R}$, strictly decreasing on $(-\infty, \gamma]$ and strictly increasing on $[\gamma, \infty)$ for some $\gamma \in \mathbb{R}$, and is coercive, i.e. $f(x) \rightarrow \infty$ as $x \rightarrow \pm \infty$.

Lemma 3.4. There exists a unique function $\varphi:(-\infty, \gamma) \rightarrow(\gamma, \infty)$ such that (3.2) admits a solution y satisfying (3.3) for $s<S$ if and only if $s<\gamma$ and $S=\varphi(s)$, in which case $y$ is unique,

$$
y^{\prime}<0 \quad \text { in } \quad(s, S) \text {, and } y^{\prime}>0 \quad \text { in }(-\infty, s) \cup(S, \infty) .
$$

Furthermore, $\varphi$ is continuous and strictly decreasing, and such that $\varphi(s) \rightarrow \gamma$ as $s \rightarrow \gamma$. 
Proof. Equation (3.2) has a unique solution satisfying $y^{\prime}(s)=0$ for any $s$. It is given by

$$
y(x)=\mathrm{e}^{-\alpha x}\left\{\mathrm{e}^{\alpha s} \frac{f(s)}{\alpha}+\int_{s}^{x} \mathrm{e}^{\alpha \eta} f(\eta) \mathrm{d} \eta\right\} .
$$

Hence, it is such that

$$
y^{\prime}(x)=\mathrm{e}^{-\alpha x}\left\{\mathrm{e}^{\alpha x} f(x)-\mathrm{e}^{\alpha s} f(s)-\alpha \int_{s}^{x} \mathrm{e}^{\alpha \eta} f(\eta) \mathrm{d} \eta\right\} .
$$

Applying the formula for integration by parts of Riemann-Stieltjes integrals, this yields

$$
y^{\prime}(x)=\mathrm{e}^{-\alpha x} \int_{s}^{x} \mathrm{e}^{\alpha \eta} \mathrm{d} f(\eta)
$$

The condition $y^{\prime}(S)=0$ consequently requires

$$
\int_{s}^{S} \mathrm{e}^{\alpha \eta} \mathrm{d} f(\eta)=0
$$

In view of the monotonicity of $f$, this necessitates $s<\gamma$ and $S=\varphi(s)>\gamma$, where

$$
\int_{\gamma}^{\varphi(s)} \mathrm{e}^{\alpha \eta} \mathrm{d} f(\eta)=-\int_{s}^{\gamma} \mathrm{e}^{\alpha \eta} \mathrm{d} f(\eta) .
$$

Because

$$
\int_{\gamma}^{z} \mathrm{e}^{\alpha \eta} \mathrm{d} f(\eta)>\int_{\gamma}^{z} \mathrm{e}^{\alpha \gamma} \mathrm{d} f(\eta)=\mathrm{e}^{\alpha \gamma}\{f(z)-f(\gamma)\} \quad \text { for } \quad z>\gamma
$$

and $f(z) \rightarrow \infty$ as $z \rightarrow \infty$, such a value $\varphi(s)$ exists for all $s<\gamma$. The identity (3.8) leads to the properties of $\varphi$. Formulae (3.6) and (3.7) together with the monotonicity of $f$ deliver (3.5).

Given (3.2) and (3.3) for $s<S$, the condition (3.4) is equivalent to

$$
f(s)=f(S)+\alpha k .
$$

Consequently, in the light of Lemma 3.4, it can be reformulated as

$$
F(s)=k,
$$

where

$$
F(s)=\frac{f(s)-f(\varphi(s))}{\alpha}=-\frac{1}{\alpha} \int_{s}^{\varphi(s)} \mathrm{d} f(\eta)
$$

for $s<\gamma$. The next lemma leads to the conclusion that (3.10) has a unique solution.

Lemma 3.5. The function $F$ is continuous, strictly decreasing, and such that $F(s) \rightarrow 0$ as $s \rightarrow \gamma$, and $F(s) \rightarrow$ $\infty$ as $s \rightarrow-\infty$.

Proof. The continuity of $F$ and its behaviour as $s \rightarrow \gamma$ follow from (3.11) and the properties of $\varphi$ provided by Lemma 3.4. To verify the remaining characteristics of $F$, take $s<r<\gamma$. By (3.11),

$$
\begin{aligned}
F(s)-F(r)= & \int_{s}^{r} \frac{\mathrm{e}^{\alpha\{\eta-\varphi(r)\}}-1}{\alpha} \mathrm{d} f(\eta)+\int_{\varphi(r)}^{\varphi(s)} \frac{\mathrm{e}^{\alpha\{\eta-\varphi(r)\}}-1}{\alpha} \mathrm{d} f(\eta) \\
& +\frac{\mathrm{e}^{-\alpha \varphi(r)}}{\alpha} \int_{r}^{\varphi(r)} \mathrm{e}^{\alpha \eta} \mathrm{d} f(\eta)-\frac{\mathrm{e}^{-\alpha \varphi(r)}}{\alpha} \int_{s}^{\varphi(s)} \mathrm{e}^{\alpha \eta} \mathrm{d} f(\eta) .
\end{aligned}
$$


The last two terms on the right-hand side of the above equality are zero by (3.8). The first two are positive by the monotonicity of the exponential function and of $f$. In fact, of the very first it can be said that

$$
\begin{aligned}
\int_{s}^{r} \frac{\mathrm{e}^{\alpha\{\eta-\varphi(r)\}}-1}{\alpha} \mathrm{d} f(\eta) & >\int_{s}^{r} \frac{\mathrm{e}^{\alpha\{r-\varphi(r)\}}-1}{\alpha} \mathrm{d} f(\eta) \\
& =\frac{1-\mathrm{e}^{\alpha\{r-\varphi(r)\}}}{\alpha}\{f(s)-f(r)\} .
\end{aligned}
$$

It follows that $F(s)>F(r)$ for all $s<r<\gamma$, and $F(s) \rightarrow \infty$ as $s \rightarrow-\infty$.

The above analysis yields the following.

Theorem 3.6. Suppose that $f$ satisfies Hypothesis 3.3. Then the QVI (2.9) has a unique solution satisfying Ansatz 3.1 .

Proof. Let $u$ be given by (3.1), where $y$ is as in Lemma 3.4. Then, $u$ is constant on $(-\infty, s]$, and, by (3.5), strictly decreasing on $[s, S]$, and strictly increasing on $[S, \infty)$. For $x \leq s$ this implies that $(M u)(x)=k+u(S)=$ $k+y(S)-y(s)+u(x)$, for $s<x<S$ it implies that $(M u)(x)=k+u(S)>k+y(S)-y(s)+u(x)$, and for $x \geq S$ it implies that $(M u)(x)=k+u(x)>u(x)$. Concurrently, $(A u)(x)=f(s)<f(x)$ for $x<s$, and $(A u)(x)=(A y)(x)=f(x)$ for $x>s$. So, if $y(s)>y(S)+k$ then $u>M u$ in $(-\infty, s)$. If $y(s)<y(S)+k$ then $(A u-f)(u-M u)>0$ in $(-\infty, s)$. Finally, if (3.4) holds then $A u<f$ and $u=M u$ in $(-\infty, s)$, and, $A u=f$ and $u<M u$ in $(s, \infty)$.

The above delivers the $(s, S)$ policy in which the optimal course of action for an inventory manager is to order up to level $S$ when the inventory level is $s$ or less, i.e. the level of shortage has reached $-s$ or more, and to freely let the inventory level decrease and shortages accrue in response to the demand otherwise.

Example 3.7. Suppose that $f$ is given by (2.5) with $p>0$ and $q>0$. Then Hypothesis 3.3 holds with $\gamma=0$, $\varphi(s)=\ln \left\{1+p\left(1-\mathrm{e}^{\alpha s}\right) / q\right\} / \alpha$, and $F(s)=-\{p s+q \varphi(s)\} / \alpha$. It follows that $s$ is the unique solution of

$$
\alpha p s+q \ln \left\{1+p\left(1-\mathrm{e}^{\alpha s}\right) / q\right\}+\alpha^{2} k=0
$$

in the interval $(-\infty, 0)$,

$$
S=-(\alpha k+p s) / q,
$$

and the function $y$ in the solution (3.1) of (2.9) satisfying Ansatz 3.1 is given by

$$
y(x)= \begin{cases}p\left[1-\alpha x-\mathrm{e}^{\alpha(s-x)}\right] / \alpha^{2} & \text { for } \quad x \leq 0 \\ q\left[\alpha x-1+\mathrm{e}^{\alpha(S-x)}\right] / \alpha^{2} & \text { for } \quad x>0 .\end{cases}
$$

The values of $s$ and $S$ in the $(s, S)$ policy naturally depend on the number $k$ and the function $f$. The ensuing corollaries of Theorem 3.6 deliver the monotonicity of this dependence.

Corollary 3.8. Let $\left(s^{ \pm}, S^{ \pm}\right)$be such that equation (3.2) has a solution $y_{ \pm}$satisfying (3.3) and (3.4) for $k=k^{ \pm}$ with $0<k^{-}<k^{+}$. Then $s^{+}<s^{-}$and $S^{-}<S^{+}$.

Proof. Recalling that $s^{ \pm}$is the unique solution of the equation $F(s)=k^{ \pm}$for $s \in(-\infty, \gamma)$, and that $F$ is strictly decreasing on $(-\infty, \gamma)$ by Lemma 3.5, necessarily $s^{+}<s^{-}$. Since $S^{ \pm}=\varphi\left(s^{ \pm}\right)$where $\varphi$ is given by Lemma 3.4, and $\varphi$ is strictly decreasing on $(-\infty, \gamma)$, this implies that $S^{+}>S^{-}$.

Corollary 3.9. Let $\left(s^{ \pm}, S^{ \pm}\right)$be such that equation (3.2) with $f$ replaced by $f^{ \pm}$has a solution $y_{ \pm}$satisfying (3.3) and (3.4), for functions $f^{ \pm}$satisfying Hypothesis 3.3 with the same number $\gamma$. If $f^{+}-f^{-}$is non-decreasing, then $s^{+} \leq s^{-}$and $S^{+} \leq S^{-}$with strict inequality in each case if $\left(f^{+}-f^{-}\right)\left(S^{-}\right)>$ $\left(f^{+}-f^{-}\right)\left(s^{+}\right)$and only if $\left(f^{+}-f^{-}\right)\left(S^{+}\right)>\left(f^{+}-f^{-}\right)\left(s^{-}\right)$. 
Proof. Let $\varphi^{ \pm}$denote the function given by Lemma 3.4, and $F^{ \pm}$the function (3.11), for $f^{ \pm}$respectively. In addition, let $S_{\infty}^{ \pm}=\lim _{\eta \rightarrow-\infty} \varphi^{ \pm}(\eta)$, and $\psi^{ \pm}$with domain $\left(\gamma, S_{\infty}^{ \pm}\right)$denote the inverse of $\varphi^{ \pm}$. By (3.8),

$$
\int_{\varphi^{+}(s)}^{\varphi^{-}(s)} \mathrm{e}^{\alpha \eta} \mathrm{d} f^{+}(\eta)=\int_{s}^{\varphi^{-}(s)} \mathrm{e}^{\alpha \eta} \mathrm{d}\left(f^{+}-f^{-}\right)(\eta)
$$

for all $s<\gamma$. So, $\varphi^{+} \leq \varphi^{-}$in $(-\infty, \gamma)$. Consequently, $S_{\infty}^{+} \leq S_{\infty}^{-}$, and $\psi^{+} \leq \psi^{-}$in $\left(\gamma, S_{\infty}^{+}\right)$. Now, by $(3.8)$ and (3.11),

$$
F^{+}(s)=\int_{s}^{\varphi^{+}(s)} \frac{\mathrm{e}^{\alpha(\eta-X)}-1}{\alpha} \mathrm{d} f^{+}(\eta)
$$

for any $s<\gamma$ and $X$. The same holds with the superscript changed from + to - . Thus, taking $X=\varphi^{+}(s)$,

$$
\begin{aligned}
F^{+}(s)-F^{-}(s)= & -\int_{s}^{\varphi^{+}(s)} \frac{1-\mathrm{e}^{\alpha\left\{\eta-\varphi^{+}(s)\right\}}}{\alpha} \mathrm{d}\left(f^{+}-f^{-}\right)(\eta) \\
& -\int_{\varphi^{+}(s)}^{\varphi^{-}(s)} \frac{\mathrm{e}^{\alpha\left\{\eta-\varphi^{+}(s)\right\}}-1}{\alpha} \mathrm{d} f^{-}(\eta) .
\end{aligned}
$$

Since $\gamma<\varphi^{+} \leq \varphi^{-}$, this implies that $F^{+} \leq F^{-}$in $(-\infty, \gamma)$. In particular, $k=F^{+}\left(s^{+}\right) \leq F^{-}\left(s^{+}\right)$. Therefore, $F^{-}\left(s^{-}\right)=k \leq F^{-}\left(s^{+}\right)$. In view of the monotonicity of $F^{-}$, this necessitates $s^{+} \leq s^{-}$. On the other hand, taking $s=\psi^{ \pm}(S)$ and $X=\psi^{-}(S)$ in (3.13) and its counterpart for $\gamma<S<S_{\infty}^{+}$,

$$
\begin{aligned}
F^{+}\left(\psi^{+}(S)\right)-F^{-}\left(\psi^{-}(S)\right)= & \int_{\psi^{-}(S)}^{S} \frac{\mathrm{e}^{\alpha\left\{\eta-\psi^{-}(S)\right\}}-1}{\alpha} \mathrm{d}\left(f^{+}-f^{-}\right)(\eta) \\
& +\int_{\psi^{+}(S)}^{\psi^{-}(S)} \frac{1-\mathrm{e}^{\alpha\left\{\eta-\psi^{-}(S)\right\}}}{\alpha} \mathrm{d} f^{+}(\eta) .
\end{aligned}
$$

Hence, $F^{+}\left(\psi^{+}\right) \geq F^{-}\left(\psi^{-}\right)$in $\left(\gamma, S_{\infty}^{+}\right)$. As a consequence, $k=F^{+}\left(\psi^{+}\left(S^{+}\right)\right) \geq F^{-}\left(\psi^{-}\left(S^{+}\right)\right)$. This implies that $F^{-}\left(\psi^{-}\left(S^{-}\right)\right)=k \geq F^{-}\left(\psi^{-}\left(S^{+}\right)\right)$, from which, in view of the monotonicity of $F^{-}$and $\psi^{-}$, it follows that $S^{+} \leq S^{-}$. It remains to establish the necessary and sufficient conditions for strictness in the inequalities $s^{+} \leq s^{-}$and $S^{+} \leq S^{-}$. Suppose first that $\left(f^{+}-f^{-}\right)\left(S^{+}\right)=\left(f^{+}-f^{-}\right)\left(s^{-}\right)$. This means that $\varphi^{+}=\varphi^{-}$in $\left[\psi^{-}\left(S^{+}\right), \gamma\right)$. In particular, $\varphi^{+}\left(\psi^{-}\left(S^{+}\right)\right)=\varphi^{-}\left(\psi^{-}\left(S^{+}\right)\right)=S^{+}$. Hence, $s^{+}=\psi^{+}\left(S^{+}\right)=\psi^{+}\left(\varphi^{+}\left(\psi^{-}\left(S^{+}\right)\right)\right)=$ $\psi^{-}\left(S^{+}\right) \geq \psi^{-}\left(S^{-}\right)=s^{-}$. Therefore, $s^{+}=s^{-}$. Consequently, $\varphi^{+}=\varphi^{-}$on $\left[s^{+}, \gamma\right)$, which in turn gives $S^{+}=\varphi^{+}\left(s^{+}\right)=\varphi^{+}\left(s^{-}\right)=\varphi^{-}\left(s^{-}\right)=S^{-}$. Suppose next that $\left(f^{+}-f^{-}\right)\left(S^{-}\right)>\left(f^{+}-f^{-}\right)\left(s^{+}\right)$. This necessitates $\left(f^{+}-f^{-}\right)\left(S^{+}\right)>\left(f^{+}-f^{-}\right)\left(s^{-}\right)$, for if this were not the case, then by what we have just proven we would have $s^{+}=s^{-}$and $S^{+}=S^{-}$, which would lead to an immediate contradiction of our supposition. However, if $\left(f^{+}-f^{-}\right)\left(S^{+}\right)>\left(f^{+}-f^{-}\right)\left(s^{-}\right)$, then $\left(f^{+}-f^{-}\right)\left(S^{-}\right)>\left(f^{+}-f^{-}\right)\left(s^{-}\right)$. Hence, (3.12) gives $S^{-}=\varphi^{-}\left(s^{-}\right)>\varphi^{+}\left(s^{-}\right)$. So, by (3.14), $F^{+}\left(s^{-}\right)>k$. Whence, $s^{+}<s^{-}$. The inequality $S^{-}>\varphi^{+}\left(s^{-}\right)$ further implies that $\psi^{+}\left(S^{-}\right)<s^{-}$. Therefore, by (3.15), $F^{+}\left(\psi^{+}\left(S^{-}\right)\right)>k$. This gives $\psi^{+}\left(S^{-}\right)<s^{+}$. Thus, $S^{+}<\varphi^{+}\left(\psi^{+}\left(S^{-}\right)\right)=S^{-}$.

Hypothesis 3.3 includes the supposition that $f(x) \rightarrow \infty$ as $x \rightarrow \infty$. The only place above where this has been used is in the proof of Lemma 3.4. Careful consideration of this usage reveals the following.

Remark 3.10. Theorem 3.6 remains valid when the supposition that $f(x) \rightarrow \infty$ as $x \rightarrow \infty$ is replaced by

$$
\int_{\gamma}^{\infty} \mathrm{e}^{\alpha \eta} \mathrm{d} f(\eta) \geq-\int_{-\infty}^{\gamma} \mathrm{e}^{\alpha \eta} \mathrm{d} f(\eta)
$$


Discounting future costs, the problem of finding an optimal control policy becomes that of finding a strategy to minimize the long-term average cost per unit time of holding and replenishing the inventory.

Remark 3.11. In the limit $\alpha \rightarrow 0$, the $(s, S)$ policy given by Theorem 3.6 converges to the classical EOQ model with shortages.

See [5] for further details, and [22,23] for the corresponding result for related problems.

\section{The Generalized $(s, S)$ POLICy FOR SEVERAL SUPPliERS}

The results on the existence and uniqueness of an $(s, S)$ policy for the inventory model with a single supplier provide a motif for the analysis of the problem with several suppliers, notwithstanding that emulation of these results turns out not to be so straightforward. We shall analyse the problem with more than one supplier in three phases. The first is to consider an ansatz that suitably generalizes the one employed for the problem with one supplier. The second is to characterize a solution satisfying this ansatz to such an extent that it becomes apparent that the QVI admits at most one such solution. The third and final phase is to prove that the characterization yields a solution of the problem, thereby obtaining an existence result to complement that of uniqueness.

\subsection{The ansatz}

As we shall duly demonstrate, the QVI (2.9) with several suppliers need not have a solution fulfilling Ansatz 3.1. The scope for finding a solution can be widened by relaxing the assumptions within the ansatz. The following involves just a minor relaxation, with far reaching consequences for the analysis.

Ansatz 4.1. The solution of (2.9) is a continuous real function $u$ such that $u=M u$ on $(-\infty, s]$, and, $u$ is differentiable, $A u=f$, and $u<M u$ in $(s, \infty)$, for some number $s$.

Ansätze 3.1 and 4.1 are equivalent for a single supplier, as is revealed by the next theorem, which expands on Lemma 3.2.

Theorem 4.2. Under Ansatz 4.1, a solution of the QVI is given by

$$
u(x)=\left\{\begin{array}{lll}
v(x) & \text { for } & x<s \\
y(x) & \text { for } & x \geq s,
\end{array}\right.
$$

where $y$ is a solution of the differential equation (3.2) satisfying

$$
y^{\prime}(s)=y^{\prime}(S)=-c_{j}
$$

and

$$
y(s)=y(S)+k_{j}+c_{j}(S-s)
$$

for some $j \in \mathcal{J}$ and $S>s$, and

$$
v(x)=\min \left\{\left(M_{\ell} y\right)(s)+c_{\ell}(s-x): \ell \in \mathcal{J}\right\} .
$$

Furthermore,

$$
y(s)=\left(M_{j} y\right)(s)<\left(M_{\ell} y\right)(s) \quad \text { for every } \ell \in \mathcal{J} \backslash\{j\}
$$

and

$$
y(x) \leq v(x) \quad \text { for } \quad x<s .
$$


We realize the proof of the above theorem through a sequence of lemmata. Throughout, $u$ will be assumed to be a given solution of (2.9) that satisfies Ansatz 4.1. We define

$$
u_{\ell}(x)=u(x)+c_{\ell} x
$$

for $\ell \in \mathcal{J}$ and $x \in \mathbb{R}$, and note that

$$
\left(M_{\ell} u\right)(x)=k_{\ell}-c_{\ell} x+\min \left\{u_{\ell}(\eta): \eta \geq x\right\} .
$$

Lemma 4.3. If $u(x)=\left(M_{\ell} u\right)(x)$ then $u_{\ell}$ has a least absolute minimum on $[x, \infty)$ at an $X>x$ such that

$$
u_{\ell}(x)=u_{\ell}(X)+k_{\ell}
$$

and

$$
u_{\ell}(z) \leq u_{\ell}(x) \quad \text { for all } z \leq X
$$

Proof. Since $\left(M_{\ell} u\right)(x)$ is well defined, $u_{\ell}$ has a least absolute minimum on $[x, \infty)$, at $X$ say, whereby $\left(M_{\ell} u\right)(x)=$ $k_{\ell}-c_{\ell} x+u_{\ell}(X)$. As $u_{\ell}(x)=\left(M_{\ell} u\right)(x)+c_{\ell} x$, this gives (4.7). Because $k_{\ell}>0$, the possibility that $X=x$ is excluded. Finally, for any $z \leq X$, by (2.9), (2.10), (4.6), and (4.7), $u(z) \leq(M u)(z) \leq\left(M_{\ell} u\right)(z) \leq k_{\ell}-c_{\ell} z+$ $u_{\ell}(X)=u_{\ell}(x)-c_{\ell} z$. Whence, (4.8).

Lemma 4.4. Suppose that $u=M u$ in the proper interval $[a, b]$. Then $u$ is concave on $[a, b]$.

Proof. Since $u$ is continuous on $\mathbb{R}$, each of the functions $u_{\ell}$ is uniformly continuous on $[a, b]$. Hence there exists a $\delta \in(0, b-a]$ such that $\left|u_{\ell}(x)-u_{\ell}(z)\right|<k_{\ell}$ for every $\ell \in \mathcal{J}$ and all $x$ and $z$ in $[a, b]$ with $|x-z| \leq \delta$. Now, let $w, x$, and $z$ be any three numbers in $[a, b]$ with $w \leq x \leq z \leq w+\delta$, and $\ell \in \mathcal{J}$ be such that $u(x)=\left(M_{\ell} u\right)(x)$. By contrivance, $u_{\ell}(x)<u_{\ell}(\eta)+k_{\ell}$ for all $x \leq \eta \leq x+\delta$. Hence, the number $X$ given by Lemma 4.3 is such that $X>x+\delta \geq w+\delta \geq z$. Consequently, by (4.8),

$$
(z-x) u_{\ell}(w)+(x-w) u_{\ell}(z) \leq(z-x) u_{\ell}(x)+(x-w) u_{\ell}(x)=(z-w) u_{\ell}(x) .
$$

Eliminating $u_{\ell}$ using (4.5) yields

$$
(z-x) u(w)+(x-w) u(z) \leq(z-w) u(x) .
$$

In view of the arbitrariness of $w, x$, and $z$, this establishes that $u$ is concave on any subinterval of $[a, b]$ of length $\delta$. Because $(a, b)$ can be covered by a finite number of such open subintervals, the concavity on the whole of $[a, b]$ follows.

Lemma 4.5. Suppose that $u=M u$ on $[a, x]$ for some $a<x$. Let $\ell$ be the least number in $\mathcal{J}$ such that $u(x)=\left(M_{\ell} u\right)(x)$. Then $u_{\ell}$ is constant and $u=M_{\ell} u$ on $[w, x]$ for some $w \in[a, x)$.

Proof. In the first instance, let $\ell \in \mathcal{J}$ be such that $u\left(w_{i}\right)=\left(M_{\ell} u\right)\left(w_{i}\right)$ for an increasing sequence $\left\{w_{i}\right\}_{i=1}^{\infty} \subset$ $[a, x)$ converging to $x$. By continuity, $u(x)=\left(M_{\ell} u\right)(x)$. By Lemma 4.4, $u_{\ell}$ is concave on $[a, x]$. Therefore, $u_{\ell}$ has a right derivative $D^{+} u_{\ell}$ at every point in $[a, x)$, and a left derivative $D^{-} u_{\ell}$ at every point in $(a, x]$, with $\left(D^{+} u_{\ell}\right)\left(\eta_{0}\right) \geq\left(D^{-} u_{\ell}\right)\left(\eta_{1}\right) \geq\left(D^{+} u_{\ell}\right)\left(\eta_{1}\right) \geq\left(D^{-} u_{\ell}\right)\left(\eta_{2}\right)$ for all $a \leq \eta_{0}<\eta_{1}<\eta_{2} \leq x$. By (4.8) applied at $x=w_{1},\left(D^{+} u_{\ell}\right)\left(w_{1}\right) \leq 0$. By $(4.8)$ applied as it is, $\left(D^{-} u_{\ell}\right)(x) \geq 0$. Hence, $\left(D^{+} u_{\ell}\right)(\eta)=\left(D^{-} u_{\ell}\right)(\eta)=0$ for all $\eta \in\left(w_{1}, x\right)$. This implies that $u_{\ell}$ is constant on $\left[w_{1}, x\right]$. Consequently, $\left(M_{\ell} u\right)(w)=k_{\ell}-c_{\ell} w+$ $\min \left\{u_{\ell}(\eta): \eta \geq w\right\}=k_{\ell}-c_{\ell} w+\min \left\{u_{\ell}(\eta): \eta \geq x\right\}=\left(M_{\ell} u\right)(x)+c_{\ell}(x-w)=u(x)+c_{\ell}(x-w)=u_{\ell}(x)-c_{\ell} w=$ $u_{\ell}(w)-c_{\ell} w=u(w)$ for all $w \in\left[w_{1}, x\right]$. If now there is an $L \in \mathcal{J} \backslash\{\ell\}$ for which $u(x)=\left(M_{L} u\right)(x)$, then by $(4.8)$ with $\ell$ replaced by $L$, there holds $\left(D^{-} u_{L}\right)(x) \geq 0$. So, $c_{L}-c_{\ell}=\left(D^{-} u_{L}-D^{-} u_{\ell}\right)(x) \geq 0$. This can be the case only if $L \geq \ell$. Consequently, $\ell$ must be the least number $L \in \mathcal{J}$ for which $u(x)=\left(M_{L} u\right)(x)$. 
Lemma 4.6. Suppose that $u=M u$ on $[x, b]$ for some $b>x$. Let $\ell$ be the greatest number in $\mathcal{J}$ such that $u(x)=\left(M_{\ell} u\right)(x)$. Then $u_{\ell}$ is constant and $u=M_{\ell} u$ on $[x, z]$ for some $z \in(x, b]$.

Proof. The proof of this lemma is analogous to that of the previous one. We omit the details.

Lemma 4.7. Let $j$ be the least number in $\mathcal{J}$ such that $u(s)=\left(M_{j} u\right)(s)$. Then $u$ is differentiable at $s$, $u^{\prime}(s)=-c_{j}$, and, $u=y$ in $[s, \infty)$, where $y$ is a solution of (3.2) that satisfies (4.1) and (4.2) for some $s<S$.

Proof. The requirement that $u$ satisfies $A u=f$ in $(s, \infty)$ is the same as asking that it is a solution of (3.2) there. Furthermore, since $f$ is continuous on $\mathbb{R}$, said solution can be extended to one, $y$ say, on $\mathbb{R}$. By Lemma 4.5, there is a $w<s$ such that $u^{\prime}=-c_{j}$ in $(w, s)$. Hence, $y^{\prime}(x)+\alpha y(x)=f(x) \geq(A u)(x)=u^{\prime}(x)+\alpha u(x)=\alpha u(x)-c_{j}$ for all $x \in(w, s)$. Passage to the limit $x \rightarrow s$ gives $y^{\prime}(s) \geq-c_{j}$. On the other hand, by Lemma $4.3, u_{j}$ has a least absolute minimum $S$ in $[s, \infty)$ such that $S>s$, and, $u_{j}(x) \leq u_{j}(s)$ for all $x \in(s, S)$. The latter inequality can be reformulated as $y(x)-y(s) \leq-c_{j}(x-s)$. Hence, dividing by $x-s$ and passing to the limit $x \rightarrow s$, we obtain $y^{\prime}(s) \leq-c_{j}$. Combining the two inequalities comparing $y^{\prime}(s)$ to $c_{j}$ yields $y^{\prime}(s)=-c_{j}$, from which it follows that $u$ is differentiable at $s$ and $u^{\prime}(s)=-c_{j}$. Because $S$ is a minimum of $u_{j}$, and $u_{j}$ is differentiable there, $u_{j}^{\prime}(S)=0$. Inasmuch $u_{j}(x)=y(x)+c_{j} x$ for $x \geq s$, this is equivalent to $y^{\prime}(S)=-c_{j}$. Thus we have obtained (4.1). The identity (4.2) follows from the observation that Lemma 4.3 additionally says that $u_{j}(S)=u_{j}(s)-k_{j}$.

Lemma 4.8. Further to Lemma 4.7, (4.4) holds.

Proof. By Ansatz 3.1, $u(s)=(M u)(s) \leq\left(M_{\ell} u\right)(s)$ for every $\ell \in \mathcal{J}$. Suppose now that $u(s)=\left(M_{\ell} u\right)(s)$ for some $\ell \in \mathcal{J} \backslash\{j\}$. Then by (4.8) at $x=s, u_{\ell}^{\prime}(s) \leq 0$. Hence, $c_{j}=-u^{\prime}(s)=c_{\ell}-u_{\ell}^{\prime}(s) \geq c_{\ell}$. This implies that $\ell<j$, which contradicts the definition of $j$ as the least number $\ell \in \mathcal{J}$ for which $u(s)=\left(M_{\ell} u\right)(s)$. Hence, necessarily, $y(s)=u(s) \leq\left(M_{\ell} u\right)(s)=\left(M_{\ell} y\right)(s)$ for every $\ell \in \mathcal{J}$ with equality if and only if $\ell=j$.

Lemma 4.9. There holds $y \leq u$ in $(-\infty, s]$.

Proof. By Lemmata 4.4-4.6, $u$ is differentiable at all but a finite number of points in $(-\infty, s)$. At an $\eta<s$ at which $u$ is differentiable,

$$
\frac{\mathrm{d}}{\mathrm{d} \eta}\left\{\mathrm{e}^{\alpha \eta} u(\eta)\right\}=\mathrm{e}^{\alpha \eta}(A u)(\eta) \leq \mathrm{e}^{\alpha \eta} f(\eta)=\mathrm{e}^{\alpha \eta}(A y)(\eta)=\frac{\mathrm{d}}{\mathrm{d} \eta}\left\{\mathrm{e}^{\alpha \eta} y(\eta)\right\}
$$

Hence, integrating piecewise, from $x<s$ to $s$, we obtain $\mathrm{e}^{\alpha s} u(s)-\mathrm{e}^{\alpha x} u(x) \leq \mathrm{e}^{\alpha s} y(s)-\mathrm{e}^{\alpha x} y(x)$. Inasmuch $u(s)=y(s)$ by Lemma 4.7 , the result follows.

Lemma 4.10. There holds $u=v$ in $(-\infty, s]$.

Proof. Let $x<s$. By (4.6), $\left(M_{\ell} u\right)(x) \leq k_{\ell}-c_{\ell} x+\min \left\{u_{\ell}(\eta): \eta \geq s\right\}=\left(M_{\ell} u\right)(s)+c_{\ell}(s-x)$ for every $\ell \in \mathcal{J}$. So, $u(x)=(M u)(x)=\min \left\{\left(M_{\ell} u\right)(x): \ell \in \mathcal{J}\right\} \leq v(x)$. Next, let $\ell$ be the greatest number in $\mathcal{J}$ such that $u(x)=\left(M_{\ell} u\right)(x)$. By Lemma 4.6, there is a $z \in(x, s]$ such that $u_{\ell}$ is constant on $[x, z]$. From the concavity of $u$ established in Lemma 4.4, it follows that $u_{\ell}$ is non-increasing on $[x, s]$. Recalling (4.6), this means that $u(x)=k_{\ell}-c_{\ell} x+\min \left\{u_{\ell}(\eta): \eta \geq s\right\}=\left(M_{\ell} u\right)(s)+c_{\ell}(s-x) \geq v(x)$. Thus we have proven that $u(x) \leq v(x)$ and $u(x) \geq v(x)$. In view of the arbitrariness of $x<s$, this ascertains that $u=v$ in $(-\infty, s)$. Lemmata 4.7 and 4.8 give $u=v$ at $s$.

Theorem 4.2 is a product of Lemmata $4.7-4.10$. 


\subsection{Uniqueness}

The basic thought behind our further analysis of the inventory model with several suppliers is that there should be an optimal $(s, S)$ policy for each of the suppliers in the absence of competitors. Thus, the function $f_{j}$ defined by

$$
f_{j}(x)=f(x)+\alpha c_{j} x \quad \text { for } \quad x \in \mathbb{R}
$$

should satisfy Hypothesis 3.3 for some number $\gamma_{j}$ for every $j \in \mathcal{J}$. In the light of (2.4) and (2.6), this necessitates

$$
\gamma_{1} \geq \gamma_{2} \geq \cdots \geq \gamma_{J}
$$

Subsequently, appealing to the theory in Section 3 , for every $j \in \mathcal{J}$ there is a unique pair $\left(s_{j}, S_{j}\right)$ with $s_{j}<$ $\gamma_{j}<S_{j}$ such that (3.2) has a solution $y_{j}$ satisfying (4.1) and (4.2) for some $s<S$ if and only if $(s, S)=\left(s_{j}, S_{j}\right)$. Furthermore, $y_{j}$ is unique,

$$
y_{j}^{\prime}<-c_{j} \quad \text { on } \quad\left(s_{j}, S_{j}\right), \quad \text { and } \quad y_{j}^{\prime}>-c_{j} \quad \text { on } \quad\left(-\infty, s_{j}\right) \cup\left(S_{j}, \infty\right) .
$$

Theorem 4.2 tells us that a solution $u$ of (2.9) satisfying Ansatz 4.1 is necessarily such that $s=s_{j}, u=y_{j}$ in $\left[s_{j}, \infty\right)$, and $y_{j} \leq u$ in $\left(-\infty, s_{j}\right)$ for some $j \in \mathcal{J}$. Consequently, our search for a solution of (2.9) can be reduced to that of finding an appropriate $j \in \mathcal{J}$.

Note that for every $j \in \mathcal{J}$ and $\ell \in \mathcal{J}$, the function $y_{j}-y_{\ell}$ is a solution of (3.2) with $f \equiv 0$. Hence, given any $\zeta \in \mathbb{R}$, there holds

$$
y_{j}(x)-y_{\ell}(x)=\left\{y_{j}(\zeta)-y_{\ell}(\zeta)\right\} \mathrm{e}^{\alpha(\zeta-x)} \quad \text { for all } \quad x \in \mathbb{R},
$$

which in turn implies that

$$
y_{j}^{\prime}(x)-y_{\ell}^{\prime}(x)=-\alpha\left\{y_{j}(\zeta)-y_{\ell}(\zeta)\right\} \mathrm{e}^{\alpha(\zeta-x)} \quad \text { for all } \quad x \in \mathbb{R} .
$$

Lemma 4.11. The number $j$ is the greatest minimizer of $y_{\ell}(\zeta)$ with respect to $\ell \in \mathcal{J}$ for all $\zeta \in \mathbb{R}$.

Proof. Pick $\zeta \in \mathbb{R}$. Suppose that $y_{\ell}(\zeta) \leq y_{j}(\zeta)$ for some $\ell \in \mathcal{J}$. Under this supposition, (4.12) gives $y_{j}^{\prime} \leq y_{\ell}^{\prime}$ in $\mathbb{R}$, with equality only if $y_{\ell}(\zeta)=y_{j}(\zeta)$. This has two consequences. Firstly,

$$
y_{j}\left(S_{\ell}\right)+y_{\ell}\left(s_{\ell}\right)-y_{\ell}\left(S_{\ell}\right)=y_{j}\left(s_{\ell}\right)+\int_{s_{\ell}}^{S_{\ell}}\left\{y_{j}^{\prime}(\eta)-y_{\ell}^{\prime}(\eta)\right\} \mathrm{d} \eta \leq y_{j}\left(s_{\ell}\right)
$$

with equality only if $y_{\ell}(\zeta)=y_{j}(\zeta)$. Secondly, if $\ell>j$, then $y_{j}^{\prime}\left(S_{\ell}\right) \leq y_{\ell}^{\prime}\left(S_{\ell}\right)=-c_{\ell}<-c_{j}$. Hence, by (4.11), $S_{\ell}>s_{j}$. On the other hand, if $\ell \leq j$, then $S_{\ell}>\gamma_{\ell} \geq \gamma_{j}>s_{j}$ by (4.10). So, $S_{\ell}>s_{j}$ irrespective of the ordering of $j$ and $\ell$. Therefore, recalling (4.6),

$$
\left(M_{\ell} u\right)\left(s_{\ell}\right) \leq k_{\ell}-c_{\ell} s_{\ell}+u_{\ell}\left(S_{\ell}\right)=y_{j}\left(S_{\ell}\right)+k_{\ell}+c_{\ell}\left(S_{\ell}-s_{\ell}\right)=y_{j}\left(S_{\ell}\right)+y_{\ell}\left(s_{\ell}\right)-y_{\ell}\left(S_{\ell}\right) .
$$

However, by Theorem 4.2,

$$
y_{j}\left(s_{\ell}\right) \leq u\left(s_{\ell}\right) \leq(M u)\left(s_{\ell}\right) \leq\left(M_{\ell} u\right)\left(s_{\ell}\right)
$$

with equality in the second inequality only if $s_{\ell} \leq s_{j}$. In which case, by $(4.11),-c_{j} \leq y_{j}^{\prime}\left(s_{\ell}\right) \leq y_{\ell}^{\prime}\left(s_{\ell}\right)=-c_{\ell}$. Hence, by (2.4), $\ell \leq j$. In combination, (4.13)-(4.15) are compatible only if they hold with equality throughout. Moreover, this necessitates $y_{\ell}(\zeta)=y_{j}(\zeta)$ and $\ell \leq j$. We conclude that for every $\ell \in \mathcal{J}$ either $y_{\ell}(\zeta)>y_{j}(\zeta)$, or, $y_{\ell}(\zeta)=y_{j}(\zeta)$ and $\ell \leq j$.

To further characterize a solution of (2.9), we have the next lemma.

Lemma 4.12. Suppose that $j$ is a minimizer of $y_{\ell}(\zeta)$ with respect to $\ell \in \mathcal{J}$ for some $\zeta \in \mathbb{R}$. Then there exists a sequence of numbers

$$
S_{j, j}=S_{j}<S_{j, j-1}<S_{j, j-2}<\cdots<S_{j, 1}
$$

such that $y_{j}^{\prime}<-c_{\ell}$ in $\left(s_{j}, S_{j, \ell}\right)$, and $y_{j}^{\prime}>-c_{\ell}$ in $\left(S_{j, \ell}, \infty\right)$ for every $\ell \in\{1,2, \ldots, j\}$. 
Proof. Pick $\ell \in\{1,2, \ldots, j\}$. By (4.12), $y_{j}^{\prime} \geq y_{\ell}^{\prime}$ in $\mathbb{R}$. Together with (4.11) for $j=\ell$, this tells us that $y_{j}^{\prime}>-c_{\ell}$ in $\left(-\infty, s_{\ell}\right) \cup\left(S_{\ell}, \infty\right)$. Hence, in view of (4.11) as it stands, we can define $s_{j, \ell}=\min \left\{x \leq s_{j}: y_{j}^{\prime}(x)=-c_{\ell}\right\} \geq s_{\ell}$ and $S_{j, \ell}=\max \left\{x \geq S_{j}: y_{j}^{\prime}(x)=-c_{\ell}\right\} \leq S_{\ell}$. Inasmuch $f_{\ell}$ satisfies Hypothesis 3.3, Lemma 3.4 says that $y_{j}^{\prime}<-c_{\ell}$ in $\left(s_{j, \ell}, S_{j, \ell}\right)$, and $y_{j}^{\prime}>-c_{\ell}$ in $\left(-\infty, s_{j, \ell}\right) \cup\left(S_{j, \ell}, \infty\right)$. The ordering of the sequence $\left\{S_{j, \ell}: \ell=1,2, \ldots, j\right\}$ follows from the continuity of $y_{j}^{\prime}$.

We are now in a position to fully identify a solution of the QVI.

Theorem 4.13. Suppose that $f_{\ell}$ satisfies Hypothesis 3.3 for every $\ell \in \mathcal{J}$. Then (2.9) has at most one solution satisfying Ansatz 4.1. To be more specific, if $u$ is such a solution, then

$$
u(x)=\left\{\begin{array}{lll}
v(x) & \text { for } & x<s_{j} \\
y_{j}(x) & \text { for } & x \geq s_{j},
\end{array}\right.
$$

where $j$ is the greatest minimizer of $y_{\ell}(\zeta)$ with respect to $\ell \in \mathcal{J}$ for all $\zeta \in \mathbb{R}$,

$$
\begin{aligned}
v(x) & =\min \left\{v_{\ell}(x): 1 \leq \ell \leq j\right\}, \\
v_{\ell}(x) & =y_{j}\left(S_{j, \ell}\right)+k_{\ell}+c_{\ell}\left(S_{j, \ell}-x\right),
\end{aligned}
$$

and $S_{j, \ell}$ is the unique number in $\left(s_{j}, \infty\right)$ for which $y_{j}^{\prime}\left(S_{j, \ell}\right)=-c_{\ell}$.

Proof. If (2.9) has a solution $u$ satisfying Ansatz 4.1, then Theorem 4.2 says that $s=s_{j}$ and $u=y_{j}$ on $[s, \infty)$ for some $j \in \mathcal{J}$. Furthermore, $u=v$ on $(-\infty, s]$ where $v$ is given by (4.3) with $y=y_{j}$. Lemma 4.11 identifies $j$ as the greatest minimizer of $y_{\ell}(\zeta)$ with respect to $\ell \in \mathcal{J}$ for all $\zeta \in \mathbb{R}$. By its definition, $v$ is piecewise linear and concave on $\mathbb{R}$. Moreover, (4.4) implies that $v=v_{j}$ in some proper interval $(w, s)$. Consequently, $\ell \in\{j+1, j+2, \ldots, J\}$ plays no role in the definition of $v$. Therefore, (4.3) reduces to (4.17) with $v_{\ell}(x)=\left(M_{\ell} y_{j}\right)\left(s_{j}\right)+c_{\ell}\left(s_{j}-x\right)$. By Lemma 4.12, $\left(M_{\ell} y_{j}\right)\left(s_{j}\right)=k_{\ell}-c_{\ell} s_{j}+\min \left\{y_{j}(\eta)+c_{\ell} \eta: \eta>s_{j}\right\}=k_{\ell}-c_{\ell} s_{j}+y_{j}\left(S_{j, \ell}\right)+c_{\ell} S_{j, \ell}$ for every $\ell \in\{1,2, \ldots, j\}$. So $v_{\ell}$ is given by (4.18) for such $\ell$.

We note that a priori, it is not possible to decide which $j \in \mathcal{J}$ may or may not minimize $y_{\ell}(\zeta)$ with respect to $\ell \in \mathcal{J}$ for all $\zeta \in \mathbb{R}$. In this regard the ordering (2.3) and the ordering (2.4) are opposing. This is borne out by the result below.

Proposition 4.14. Let $y_{ \pm}$be solutions of equation (3.2) satisfying (4.1) and (4.2) with $s, S, c_{j}$, and $k_{j}$ replaced by $s^{ \pm}, S^{ \pm}, c^{ \pm}$, and $k^{ \pm}$respectively. Define $f^{ \pm}(x)=f(x)+\alpha c^{ \pm} x$ for $x \in \mathbb{R}$, and suppose that $f^{ \pm}$satisfy Hypothesis 3.3 with the same number $\gamma$. If $c^{+} \geq c^{-}, k^{+} \geq k^{-}$, and $c^{+}+k^{+}>c^{-}+k^{-}$, then $y_{+}>y_{-}$in $\mathbb{R}$.

Proof. Fix $x \in \mathbb{R}$. By the equivalence of the QVI for a single supplier with arbitrary $c$ and with $c=0$ outlined at the start of Section 3, (3.2), and (3.6),

$$
\alpha y_{ \pm}(x)-f(x)=-y_{ \pm}^{\prime}(x)=c^{ \pm}-\int_{s^{ \pm}}^{x} \mathrm{e}^{\alpha(\eta-x)} \mathrm{d} f^{ \pm}(\eta) .
$$

So,

$$
\begin{aligned}
\alpha\left\{y_{+}(x)-y_{-}(x)\right\} & =c^{+}-c^{-}-\int_{s^{+}}^{x} \mathrm{e}^{\alpha(\eta-x)} \mathrm{d}\left(f^{+}-f^{-}\right)(\eta)-\int_{s^{+}}^{s^{-}} \mathrm{e}^{\alpha(\eta-x)} \mathrm{d} f^{-}(\eta) \\
& =\left(c^{+}-c^{-}\right) \mathrm{e}^{\alpha\left(s^{+}-x\right)}-\int_{s^{+}}^{s^{-}} \mathrm{e}^{\alpha(\eta-x)} \mathrm{d} f^{-}(\eta) .
\end{aligned}
$$

By Corollaries 3.8 and $3.9, s^{+}<s^{-}<\gamma$, which implies that the above expression is positive. Hence $y_{+}(x)>$ $y_{-}(x)$. 


\subsection{Existence}

Having shown that a solution of the QVI satisfying Ansatz 4.1 is necessarily given by (4.16)-(4.18), where $j$ is the greatest minimizer of $y_{\ell}(\zeta)$ with respect to $\ell \in \mathcal{J}$ for all $\zeta \in \mathbb{R}$, the task of proving existence is somewhat eased. It suffices to show that the function $u$ so defined is what we seek. To accomplish this, it will be advantageous to first characterize said function in a little more detail.

Let

$$
\sigma_{1}=s_{j}>\sigma_{2}>\cdots>\sigma_{N}
$$

be such that $v$ is affine on each of the intervals

$$
I_{1}=\left(\sigma_{2}, \sigma_{1}\right), I_{2}=\left(\sigma_{3}, \sigma_{2}\right), \ldots, I_{N}=\left(-\infty, \sigma_{N}\right)
$$

and not on the union of any two of them. Set

$$
\mathcal{M}=\{1,2 \ldots, N\}
$$

and define $\kappa: \mathcal{M} \rightarrow\{1,2, \ldots, j\}$ by

$$
v=v_{\kappa(m)} \text { on } I_{m}
$$

When $N \geq 2, u$ will not be differentiable at $\sigma_{m}$ for $m \in\{2,3, \ldots, N\}$, irrespective of its differentiability at $s_{j}$ in accordance with Lemma 4.7. Thus, $A u$ is not well defined there. We subsequently interpret the condition $A u \leq f$ in $(-\infty, s)$ as $A u \leq f$ in $I_{m}$ for every $m \in \mathcal{M}$. With this understanding, we formulate our existence result.

Theorem 4.15. Suppose that $f_{\ell}$ satisfies Hypothesis 3.3 for every $\ell \in \mathcal{J}$, and $j$ is the greatest minimizer of $y_{\ell}(\zeta)$ with respect to $\ell \in \mathcal{J}$ for some $\zeta \in \mathbb{R}$. Then the function u given by (4.16)-(4.18) satisfies Ansatz 4.1. Moreover, if $j=1$, then $u$ solves (2.9) and satisfies Ansatz 3.1. On the other hand, if $j \geq 2$, then $N \geq 2$, and $u$ solves (2.9) if and only if

$$
\alpha v_{\kappa(m)}\left(\sigma_{m}\right) \leq f\left(\sigma_{m}\right)+c_{\kappa(m)} \quad \text { for every } m \in\{2,3, \ldots, N\} .
$$

The above theorem will be proven with the aid of four further lemmata.

Lemma 4.16. Suppose that $j$ is as stated in Theorem 4.15. Then $\left(M_{\ell} y_{j}\right)(x)$ is well defined for all $x \in \mathbb{R}$ and every $\ell \in \mathcal{J}$. Moreover, $y_{j}(x) \leq\left(M_{\ell} y_{j}\right)(x)$ for all $x \geq s_{j}$ with equality if and only if $x=s_{j}$ and $\ell=j$.

Proof. Fix $\ell \in \mathcal{J}$. Lemma 4.12 states that there is a number $S_{j, 1}$ such that $y_{j}^{\prime}(\eta)>-c_{1}$ for all $\eta>S_{j, 1}$. Consequently, $y_{j}^{\prime}(\eta)>-c_{\ell}$ for all such $\eta$. It follows that $\eta \mapsto y_{j}(\eta)+c_{\ell} \eta$ has an absolute minimum in $[x, \infty)$ for all $x \in \mathbb{R}$. Thus $\left(M_{\ell} y_{j}\right)(x)$ is well defined for all such $x$. By (4.11) with $j=\ell, \eta \mapsto y_{\ell}(\eta)+c_{\ell} \eta$ is strictly increasing on $\left(-\infty, s_{\ell}\right]$, strictly decreasing on $\left[s_{\ell}, S_{\ell}\right]$, and strictly increasing on $\left[S_{\ell}, \infty\right)$. Therefore,

$$
\left\{y_{\ell}(x)+c_{\ell} x\right\}-\left\{y_{\ell}(z)+c_{\ell} z\right\} \leq\left\{y_{\ell}\left(s_{\ell}\right)+c_{\ell} s_{\ell}\right\}-\left\{y_{\ell}\left(S_{\ell}\right)+c_{\ell} S_{\ell}\right\}=k_{\ell}
$$

for all $x \leq z$, with equality if and only if $x=s_{\ell}$ and $z=S_{\ell}$. However, by (4.12),

$$
y_{j}(x)-y_{j}(z)=y_{\ell}(x)-y_{\ell}(z)-\int_{x}^{z}\left\{y_{j}^{\prime}(\eta)-y_{\ell}^{\prime}(\eta)\right\} \mathrm{d} \eta \leq y_{\ell}(x)-y_{\ell}(z),
$$

with equality if and only if $y_{j}(\zeta)=y_{\ell}(\zeta)$ or $z=x$. Combining (4.24) and (4.25), we deduce that

$$
y_{j}(x)-y_{j}(z) \leq k_{\ell}+c_{\ell}(z-x) \text { for all } x \leq z,
$$

with equality if and only if $x=s_{\ell}, z=S_{\ell}$, and $y_{j}(\zeta)=y_{\ell}(\zeta)$. It follows that $y_{j}(x) \leq\left(M_{\ell} y_{j}\right)(x)$ for all $x \geq s_{j}$, with equality if and only if $x=s_{\ell}$ and $y_{j}(\zeta)=y_{\ell}(\zeta)$. The latter criterion implies that $y_{j} \equiv y_{\ell}$. The former implies that $s_{\ell} \geq s_{j}$. Subsequently, recalling that (4.11) with $j=\ell$ holds, we have $c_{\ell} \geq-y_{\ell}^{\prime}\left(s_{j}\right)=-y_{j}^{\prime}\left(s_{j}\right)=c_{j}$. Thus, $\ell \geq j$. However, $j$ is the greatest minimizer of $y_{\ell}(\zeta)$ with respect to $\ell \in \mathcal{J}$. We are therefore forced to conclude that $y_{j}(x) \leq\left(M_{\ell} y_{j}\right)(x)$ for all $x \geq s_{j}$ with equality as in the statement of the lemma. 
Lemma 4.17. Further to Lemma 4.16, the function u defined by (4.16) to (4.18) is continuous, and continuously differentiable everywhere except at $\sigma_{m}$ for $m \in\{2,3, \ldots, N\}$ when $N \geq 2$.

Proof. Lemma 4.16 implies that

$$
u\left(s_{j}\right)=\left(M_{j} u\right)\left(s_{j}\right)<\left(M_{\ell} u\right)\left(s_{j}\right) \quad \text { for every } \ell \in\{1,2, \ldots, j\} .
$$

By Lemma $4.12,\left(M_{\ell} u\right)\left(s_{j}\right)=v_{\ell}\left(s_{j}\right)$, where $v_{\ell}$ is defined by (4.18), for every such $\ell$. By its definition (4.17), $v$ is piecewise linear and concave on $\mathbb{R}$. Thus the numbers (4.19), the intervals (4.20), and the set (4.21) are well defined. Moreover, (4.22) defines a strictly decreasing function $\kappa: \mathcal{M} \rightarrow \mathcal{J}$. Noting that (4.26) implies that $v=v_{j}$ in $I_{1}$, and that $\left(c_{1}-c_{\ell}\right)\left(s_{j}-x\right) \rightarrow-\infty$ as $x \rightarrow-\infty$ for every $\ell \in\{2,3, \ldots, j\}$, necessarily

$$
\kappa(1)=j \quad \text { and } \quad \kappa(N)=1 .
$$

Because $v=v_{j}$ and therefore $v^{\prime}=-c_{j}$ on $I_{1}$, and $y_{j}^{\prime}\left(s_{j}\right)=-c_{j}, u$ is differentiable at $s_{j}$. Hence, $u$ is continuous in $\mathbb{R}$, and continuously differentiable as stated.

Lemma 4.18. There holds $v=M u$ on $\left(-\infty, s_{j}\right]$.

Proof. We prove that $v=M u$ on $\overline{I_{m}}$ by induction on $m$. Our induction hypothesis is

$$
\left(M_{\ell} u\right)\left(\sigma_{m}\right)=v_{\ell}\left(\sigma_{m}\right) \text { for } 1 \leq \ell \leq \kappa(m),
$$

and

$$
(M u)\left(\sigma_{m}\right)=v\left(\sigma_{m}\right) .
$$

Recalling that $\sigma_{1}=s_{j}$, for $m=1$ (4.28) is a tautology, and (4.29) is true by Lemma 4.16. Suppose that (4.28) and (4.29) are true for arbitrary $m \in \mathcal{M}$. Fix $x \in \overline{I_{m}}$. For $1 \leq \ell \leq \kappa(m)$, resurrecting the notation (4.5), $u_{\ell}$ is nonincreasing on $I_{m}$. Hence, $\left(M_{\ell} u\right)(x)=\left(M_{\ell} u\right)\left(\sigma_{m}\right)+c_{\ell}\left(\sigma_{m}-x\right)$. Substituting (4.28) gives $\left(M_{\ell} u\right)(x)=v_{\ell}\left(\sigma_{m}\right)+$ $c_{\ell}\left(\sigma_{m}-x\right)=v_{\ell}(x) \geq v(x)$, with equality for $\ell=\kappa(m)$. On the other hand, for $\kappa(m) \leq \ell \leq J, u_{\ell}$ is nondecreasing on $I_{m}$. Hence, it has an absolute minimum in $[x, \infty)$ at $x$, or in $\left[\sigma_{m}, \infty\right)$. In the first instance, $\left(M_{\ell} u\right)(x)=$ $k_{\ell}+u(x)$. In the second instance, $\left(M_{\ell} u\right)(x)=\left(M_{\ell} u\right)\left(\sigma_{m}\right)+c_{\ell}\left(\sigma_{m}-x\right) \geq(M u)\left(\sigma_{m}\right)+c_{\kappa(m)}\left(\sigma_{m}-x\right)$. So by (4.29), $\left(M_{\ell} u\right)(x) \geq v\left(\sigma_{m}\right)+c_{\kappa(m)}\left(\sigma_{m}-x\right)=v_{\kappa(m)}\left(\sigma_{m}\right)+c_{\kappa(m)}\left(\sigma_{m}-x\right)=v_{\kappa(m)}(x)$. Therefore, in both instances, $\left(M_{\ell} u\right)(x) \geq v(x)$. Altogether this implies that $\left(M_{\ell} u\right)(x)=v_{\ell}(x)$ for every $\ell \in\{1,2, \ldots, \kappa(m)\}$, and $(M u)(x)=v(x)$. Thus, $M u=v$ in $\overline{I_{m}}$, and, (4.28) and (4.29) hold with $m$ replaced by $m+1$ when $m \leq N-1$.

Lemma 4.19. There holds $A u \leq f$ in $\left(-\infty, s_{j}\right)$ if and only if $(4.23)$ is true.

Proof. Let $m \in \mathcal{M}$. Recalling the notation (4.9), for $x \in I_{m}$,

$$
\begin{aligned}
(A u-f)(x) & =\left(A v_{\kappa(m)}-f\right)(x)=\alpha v_{\kappa(m)}(x)-c_{\kappa(m)}-f(x) \\
& =\alpha v_{\kappa(m)}\left(\sigma_{m}\right)-f\left(\sigma_{m}\right)-c_{\kappa(m)}+f_{\kappa(m)}(x)-f_{\kappa(m)}\left(\sigma_{m}\right) .
\end{aligned}
$$

Since $\sigma_{m} \leq s_{j}<\gamma_{j} \leq \gamma_{\ell}$ and $f_{\ell}$ is strictly decreasing on $\left(-\infty, \gamma_{\ell}\right]$ for every $\ell \in\{1,2 \ldots, j\}$, it follows that $A u-f \leq 0$ in $I_{m}$ if and only if $\alpha v_{\kappa(m)}\left(\sigma_{m}\right) \leq f_{\kappa(m)}\left(\sigma_{m}\right)+c_{\kappa(m)}$. However, $\alpha v_{\kappa(1)}\left(\sigma_{1}\right)=\alpha v\left(s_{j}\right)=\alpha y_{j}\left(s_{j}\right)=$ $f\left(s_{j}\right)-y_{j}^{\prime}\left(s_{j}\right)=f\left(s_{j}\right)+c_{j}=f\left(\sigma_{1}\right)+c_{\kappa(1)}$. So, the inequality can be discounted for $m=1$.

Given the above four lemmata, verification of Theorem 4.15 is a sinecure.

Proof of Theorem 4.15. Since $y_{j}$ solves (3.2), and $u=y_{j}$ in $\left[s_{j}, \infty\right), A u=f$ there. Inasmuch $j$ is the greatest minimizer of $\left\{y_{\ell}(\zeta): \ell \in \mathcal{J}\right\}$, Lemma 4.16 states that $u<M u$ in $\left(s_{j}, \infty\right)$ and $u=M u$ at $s_{j}$. Hereupon, Lemma 4.17 implies that $u$ has the regularity set out in Ansatz 4.1. By Lemma 4.18, $u=M u$ in $\left(-\infty, s_{j}\right]$. Thus, $u$ satisfies Ansatz 4.1 in full. Plainly, it satisfies Ansatz 3.1 if and only if $N=1$. By (4.27), $N=1$ if and only if $j=1$. To conclude whether $u$ solves (2.9), it remains to ascertain whether $A u \leq f$ in $\left(-\infty, s_{j}\right)$. By Lemma 4.19, this will be the case if and only if $N=1$, or, $N \geq 2$ and (4.23) holds. 
Together Theorems 4.13 and 4.15 reveal a computational procedure for finding a solution of the QVI.

Corollary 4.20. If it exists, the unique solution $u$ of the QVI (2.9) satisfying Ansatz 4.1 is obtainable as follows. Let $s_{\ell}<S_{\ell}$ be the unique so-ordered solution pair of the simultaneous equations

$$
\int_{s_{\ell}}^{S_{\ell}} \mathrm{e}^{\alpha \eta} \mathrm{d} f_{\ell}(\eta)=0 \quad \text { and } \quad f_{\ell}\left(s_{\ell}\right)=f_{\ell}\left(S_{\ell}\right)+k_{\ell}
$$

and set

$$
Y_{\ell}=f(0)+c_{\ell}-\int_{s_{\ell}}^{0} \mathrm{e}^{\alpha \eta} \mathrm{d} f_{\ell}(\eta)
$$

for $\ell \in \mathcal{J}$. Let $j$ be the largest number in $\mathcal{J}$ with the property that $Y_{\ell} \geq Y_{j}$ for every $\ell \in \mathcal{J}$. Define

$$
B_{j}=\left\{f_{j}\left(s_{j}\right)+c_{j}\right\} / \alpha .
$$

If $j=1$ then

$$
u(x)=\mathrm{e}^{-\alpha x}\left\{\mathrm{e}^{\alpha s_{j}} \frac{f\left(s_{j}\right)+c_{j}}{\alpha}+\int_{s_{j}}^{x} \mathrm{e}^{\alpha \eta} f(\eta) \mathrm{d} \eta\right\} \quad \text { for } \quad x \geq s_{j},
$$

and

$$
u(x)=B_{1}-c_{1} x \quad \text { for } \quad x<s_{j} .
$$

If $j \geq 2$, then for every $\ell \in\{1,2, \ldots, j-1\}$, let

$$
B_{\ell}=k_{\ell}+\left\{f_{\ell}\left(S_{j, \ell}\right)+c_{\ell}\right\} / \alpha
$$

where $S_{j, \ell}$ is the unique number in $\left(S_{j}, \infty\right)$ for which

$$
\int_{S_{j}}^{S_{j, \ell}} \mathrm{e}^{\alpha \eta} \mathrm{d} f_{j}(\eta)=\left(c_{j}-c_{\ell}\right) \mathrm{e}^{\alpha S_{j, \ell}} .
$$

Set $\sigma_{1}=s_{j}$ and $\kappa(1)=j$. By induction on $m \geq 2$, define $\kappa(m)$ as the least maximizer and $\sigma_{m}$ as the maximum value of $\left(B_{\kappa(m-1)}-B_{\ell}\right) /\left(c_{\kappa(m-1)}-c_{\ell}\right)$ with respect to $\ell \in\{1,2, \ldots, \kappa(m-1)-1\}$, and

$$
T_{m}=f_{\kappa(m)}\left(\sigma_{m}\right)+c_{\kappa(m)}-\alpha B_{\kappa(m)} .
$$

If $T_{m}<0$, then (2.9) has no solution satisfying Ansatz 4.1. Otherwise, continue the induction process, ending when $\kappa(m)=1$ and $T_{m} \geq 0$. The number $m$ at this stage is $N$. Hereupon, $u$ is given by (4.33),

$$
u(x)=B_{\kappa(m)}-c_{\kappa(m)} x \quad \text { for } \quad \sigma_{m+1} \leq x<\sigma_{m} \quad \text { and } \quad 1 \leq m \leq N-1,
$$

and

$$
u(x)=B_{1}-c_{1} x \quad \text { for } \quad x<\sigma_{N} .
$$

Proof. By the theory in Section $3, s_{\ell}$ and $S_{\ell}$ satisfy (3.7) and (3.9) with $f$ and $k$ replaced by $f_{\ell}$ and $k_{\ell}$ respectively for every $\ell \in \mathcal{J}$. In other words, they are given by (4.30). Furthermore, by (3.6),

$$
y_{\ell}^{\prime}(x)+c_{\ell}=\int_{s_{\ell}}^{x} \mathrm{e}^{\alpha(\eta-x)} \mathrm{d} f_{\ell}(\eta) \quad \text { for all } \quad x \in \mathbb{R} .
$$

So, by (3.2), $y_{\ell}(0)=Y_{\ell} / \alpha$. With $j$ determined, (4.30) and (4.36) with $\ell=j$ imply that the unique number $S_{j, \ell}$ in $\left(s_{j}, \infty\right)$ for which $y_{j}^{\prime}\left(S_{j, \ell}\right)=-c_{\ell}$ is given by (4.35) for every $\ell \in\{1,2, \ldots, j\}$. Writing (4.18) as

$$
v_{\ell}(x)=B_{\ell}-c_{\ell} x,
$$


there holds $B_{\ell}=y_{j}\left(S_{j, \ell}\right)+k_{\ell}+c_{\ell} S_{j, \ell}=\left\{f\left(S_{j, \ell}\right)-y_{j}^{\prime}\left(S_{j, \ell}\right)\right\} / \alpha+k_{\ell}+c_{\ell} S_{j, \ell}=\left\{f\left(S_{j, \ell}\right)+c_{\ell}\right\} / \alpha+k_{\ell}+c_{\ell} S_{j, \ell}$. Thus, $B_{\ell}$ is given by (4.34) for every $\ell \in\{1,2, \ldots, j\}$. In the particular case $\ell=j$, as $f_{j}\left(S_{j, j}\right)=f_{j}\left(S_{j}\right)=$ $f_{j}\left(s_{j}\right)+\alpha k_{j}$, (4.34) simplifies to (4.32). Herewith all the ingredients of Theorems 4.13 and 4.15 have been assembled. The induction process extracts $v$ from (4.17) and (4.37). The condition (4.23) translates as $T_{m} \geq 0$ for every $m \in\{2,3, \ldots, N\}$. Explicit solution of (3.2) under the condition $y_{j}^{\prime}\left(s_{j}\right)=-c_{j}$ delivers (4.33).

Example 4.21. Suppose that $f$ is given by (2.5) with $p>\alpha c_{J}>\alpha c_{1}>-q$, so that $f_{\ell}$ satisfies Hypothesis 3.3 with $\gamma_{\ell}=0$ for every $\ell \in \mathcal{J}$. Then Example 3.7 tells us that $s_{\ell}$ is the unique solution of

$$
\alpha\left(p-\alpha c_{\ell}\right) s_{\ell}+\left(q+\alpha c_{\ell}\right) \ln \left\{1+\left(p-\alpha c_{\ell}\right)\left(1-\mathrm{e}^{\alpha s_{\ell}}\right) /\left(q+\alpha c_{\ell}\right)\right\}+\alpha^{2} k_{\ell}=0
$$

in the interval $(-\infty, 0)$, for every $\ell \in \mathcal{J}$. Furthermore,

$$
S_{j}=-\left\{\alpha k_{j}+\left(p-\alpha c_{j}\right) s_{j}\right\} /\left(q+\alpha c_{j}\right)
$$

and

$$
u(x)= \begin{cases}{\left[p(1-\alpha x)-\left(p-\alpha c_{j}\right) \mathrm{e}^{\alpha\left(s_{j}-x\right)}\right] / \alpha^{2}} & \text { for } \quad s_{j} \leq x \leq 0 \\ {\left[q(\alpha x-1)+\left(q+\alpha c_{j}\right) \mathrm{e}^{\alpha\left(S_{j}-x\right)}\right] / \alpha^{2}} & \text { for } \quad x>0\end{cases}
$$

By (4.31),

$$
Y_{\ell}=\left\{p-\left(p-\alpha c_{\ell}\right) \mathrm{e}^{\alpha s_{\ell}}\right\} / \alpha
$$

By (4.32),

$$
B_{j}=\left\{c_{j}-\left(p-\alpha c_{j}\right) s_{j}\right\} / \alpha
$$

By (4.35),

$$
S_{j, \ell}=S_{j}+\left\{\ln \left(q+\alpha c_{j}\right)-\ln \left(q+\alpha c_{\ell}\right)\right\} / \alpha
$$

and by (4.34),

$$
B_{\ell}=k_{\ell}+\left\{\left(q+\alpha c_{\ell}\right) S_{j, \ell}+c_{\ell}\right\} / \alpha
$$

for every $\ell \in\{1,2, \ldots, j-1\}$. With the further details as in the statement of Corollary 4.20,

$$
T_{m}=c_{\kappa(m)}-B_{\kappa(m)}-\left\{p-\alpha c_{\kappa(m)}\right\} \sigma_{m} \quad \text { for } \quad 2 \leq m \leq N .
$$

Example 4.22. Taking $p=q=3, \alpha=1, J=2, k_{1}=2, k_{2}=1, c_{1}=1$, and $c_{2}=2$, numerical calculation with propriety software gives $s_{1} \approx-1.683, s_{2} \approx-1.767, Y_{1} \approx 2.628$, and $Y_{2} \approx 2.829$. Thereafter, $S_{1} \approx 0.342$. So $j=1$ and the QVI has a unique solution satisfying Ansatz 3.1. This means that the optimal control is an $(s, S)$ policy involving supplier 1 only, whereby $s=s_{1}$ and $S=S_{1}$.

Example 4.23. Substitution of $k_{1}=6$ in Example 4.22 gives similarly $s_{1} \approx-3.796, Y_{1} \approx 2.955$, and $s_{2}$ and $Y_{2}$ as in that example. Thereafter, $S_{2} \approx 0.153, S_{2,1} \approx 0.377, B_{1} \approx 8.506, B_{2} \approx 3.767, \sigma_{2} \approx-4.739$, and $T_{2} \approx 1.972$. So $j=2$ and the QVI has a unique solution satisfying Ansatz 4.1 that does not satisfy Ansatz 3.1. This means that the optimal control is a generalized $(s, S)$ policy, whereby one does not intervene if the inventory level $x$ is greater than $s_{2}$, one orders from supplier 2 up to the level $S_{2}$ if $\sigma_{2}<x \leq s_{2}$, and one orders from supplier 1 up to the level $S_{2,1}$ if $x<\sigma_{2}$.

Example 4.24. Substitution of $k_{1}=4$ in Example 4.22 gives $s_{1} \approx-2.769, Y_{1} \approx 2.875$, and $s_{2}$ and $Y_{2}$ as in that example. Thereafter, $S_{2}, S_{2,1}$, and $B_{2}$ are as in Example $4.23, B_{1} \approx 6.506, \sigma_{2} \approx-2.739$, and $T_{2} \approx-0.028$. So the QVI has no solution satisfying Ansatz 4.1, and no optimal control in the form of a generalized $(s, S)$ policy. 
Examples 4.22 to 4.24 show that the three essentially different outcomes of Theorem 4.15 are each viable.

From Theorem 4.15 and the above examples, we deduce that the inventory control problem with several suppliers may or may not admit a generalized $(s, S)$ policy as an optimal solution. If it does, there is only one such a policy. This policy will be a conventional $(s, S)$ policy if and only if it is to order solely from the first supplier, i.e. the supplier with the least cost per unit item. Otherwise, there will be a unique generalized $(s, S)$ policy, which can be explained in terms of the above theory by defining $\boldsymbol{S}_{1}=S_{j, j}=S_{j}$, and $\boldsymbol{S}_{m}=S_{j, \kappa(m)}$ for $2 \leq m \leq N$. Necessarily

$$
\sigma_{N}<\sigma_{N-1}<\cdots<\sigma_{1}<\boldsymbol{S}_{1}<\boldsymbol{S}_{2}<\cdots<\boldsymbol{S}_{N} .
$$

The strategy of an inventory manager with inventory level $x$ is as follows. If $x>\sigma_{1}$ then do not intervene. If $\sigma_{2}<x \leq \sigma_{1}$ then order to the level $\boldsymbol{S}_{1}$ from supplier $j$. If $\sigma_{m+1}<x<\sigma_{m}$ for some $2 \leq m \leq N-1$ then order to the level $\boldsymbol{S}_{m}$ from supplier $\kappa(m)$. If $x<\boldsymbol{s}_{N}$ then order to the level $\boldsymbol{S}_{N}$ from supplier 1. Suppliers $j+1$, $j+2, \ldots, J$ are excluded from this policy, as may be supplier $\ell$ for $\ell \in\{\kappa(m)+1, \kappa(m)+2, \ldots, \kappa(m-1)-1\}$ when $\kappa(m) \leq \kappa(m-1)-2$ for some $m \in\{2,3, \ldots, N\}$. If $x=\sigma_{m}$ for such $m$ then the manager could order to the level $\boldsymbol{S}_{m-1}$ from supplier $\kappa(m-1)$ or to the level $\boldsymbol{S}_{m}$ from supplier $\kappa(m)$. In fact, from the construction of the solution $u$ of the QVI, a point $\left(\sigma_{m}, u\left(\sigma_{m}\right)\right)$, at which the slope of the graph of $u$ changes, could conceivably be a point of intersection of the graphs of more than two functions $v_{\ell}$. Supposing that such a point is common to the graphs of $v_{\ell}$ for $\ell \in \mathcal{L}$, where $\{\kappa(m), \kappa(m-1)\} \subset \mathcal{L} \subseteq\{\kappa(m), \kappa(m)+1, \ldots, \kappa(m-1)\}$, the possibility is open to place an order from the inventory level $\sigma_{m}$ to the level $S_{j, \ell}$ for every $\ell \in \mathcal{L}$.

The aforementioned features of a generalized $(s, S)$ policy are substantiated by the following.

Example 4.25. Suppose that $\alpha=1$ and $f$ is given by (2.5) with $p=q=9$. Take $J=4, k_{1}=14, k_{2}=12$, $k_{3}=3, k_{4}=2, c_{1}=3, c_{2}=4, c_{3}=5$, and $c_{4}=6$. Pursuant to Example 4.21, $s_{1} \approx-3.114, s_{2} \approx-3.217$, $s_{3} \approx-1.440, s_{4} \approx-1.360, Y_{1} \approx 8.734, Y_{2} \approx 8.800, Y_{3} \approx 8.053$, and $Y_{4} \approx 8.230$. So, $j=3, \kappa(1)=3$, and $\sigma_{1}=s_{3}$. Hereafter, it can be ascertained that $S_{3} \approx 0.197, S_{3,2} \approx 0.271, S_{3,1} \approx 0.351, B_{1} \approx 21.22, B_{2} \approx 19.53$, and $B_{3} \approx 10.76$, leading to $\left(B_{3}-B_{1}\right) /\left(c_{3}-c_{1}\right) \approx-5.228>\left(B_{3}-B_{2}\right) /\left(c_{3}-c_{2}\right) \approx-8.766$. Thus, $\kappa(2)=1$, $\sigma_{2}=\left(B_{3}-B_{1}\right) /\left(c_{3}-c_{1}\right)$, and $T_{2} \approx 13.15$. There is a unique generalized $(s, S)$ policy, with $N=2, \boldsymbol{S}_{1}=S_{3}$, and $\boldsymbol{S}_{2}=S_{3,1}$.

Despite there being four suppliers available in Example 4.25, the optimal inventory policy involves only suppliers 1 and 3. This illustrates that available suppliers may be excluded from the optimal policy (when it exists), and, that this exclusion need not be confined to suppliers $j+1, j+2, \ldots, J$, where supplier $j$ is the last supplier included. As a counterbalance to this example, the next, with the same number of available suppliers, shows that it is possible that every supplier is included.

Example 4.26. Let $\alpha, f, J$, and $c_{\ell}$ for $1 \leq \ell \leq 4$ be as in Example 4.25 . Take $k_{1}=18, k_{2}=11, k_{3}=6$, and $k_{4}=3$. Employing the adopted computational strategy, $s_{1} \approx-3.796, s_{2} \approx-3.010, s_{3} \approx-2.301, s_{4} \approx-1.767$, $Y_{1} \approx 8.865, Y_{2} \approx 8.754, Y_{3} \approx 8.599$, and $Y_{4} \approx 8.488$. So, $j=4, \kappa(1)=4$, and $\sigma_{1}=s_{4}$. Then, $S_{4,4}=S_{4} \approx 0.153$, $S_{4,3} \approx 0.222, S_{4,2} \approx 0.297, S_{4,1} \approx 0.377, B_{1} \approx 25.52, B_{2} \approx 18.86, B_{3} \approx 14.11$, and $B_{4} \approx 11.30$, leading to $\left(B_{4}-B_{3}\right) /\left(c_{4}-c_{3}\right) \approx-2.812>\left(B_{4}-B_{2}\right) /\left(c_{4}-c_{2}\right) \approx-3.777>\left(B_{4}-B_{1}\right) /\left(c_{4}-c_{1}\right) \approx-4.739$. Thus, $\kappa(2)=3, \sigma_{2}=\left(B_{4}-B_{3}\right) /\left(c_{4}-c_{3}\right)$, and $T_{2} \approx 2.136$. Thereafter, $\left(B_{3}-B_{2}\right) /\left(c_{3}-c_{2}\right) \approx-4.741>$ $\left(B_{3}-B_{1}\right) /\left(c_{3}-c_{1}\right) \approx-5.702$. So, $\kappa(3)=2, \sigma_{3}=\left(B_{3}-B_{2}\right) /\left(c_{3}-c_{2}\right)$, and $T_{3} \approx 8.850$. Finally, $\kappa(4)=1$, $\sigma_{4}=\left(B_{2}-B_{1}\right) /\left(c_{2}-c_{1}\right) \approx-6.664$, and $T_{4} \approx 17.46$. There is a unique generalized $(s, S)$ policy, with $N=4$ and $\boldsymbol{S}_{m}=S_{4, \kappa(m)}=S_{4,5-m}$ for $1 \leq m \leq 4$.

The optimal policies in Examples 4.25 and 4.26 are both such that for every inventory level $x=\sigma_{m}$, where $2 \leq m \leq N$, a manager could order to the level $\boldsymbol{S}_{\kappa(m-1)}$ from supplier $\kappa(m-1)$, or to the level $\boldsymbol{S}_{\kappa(m)}$ from supplier $\kappa(m)$. Our final example, however improbable that its like would occur in practice, confirms that an optimal policy could verily be such that it contains an inventory level $\sigma_{m}$ from which one could order to the level $S_{j, \ell}$ for a supplier $\ell$ supplementary to the obligatory $\kappa(m-1)$ and $\kappa(m)$. 
Example 4.27. Let $\alpha, f, J, k_{1}, k_{4}$, and $c_{\ell}$ for $1 \leq \ell \leq 4$ be as in Example 4.26. With $s_{4}, Y_{1}, Y_{4}, S_{4, \ell}$ for $1 \leq \ell \leq 4, B_{1}$, and $B_{4}$ as in that example, set $B_{\ell}=B_{4}-\left(B_{4}-B_{1}\right)\left(c_{4}-c_{\ell}\right) /\left(c_{4}-c_{1}\right)$ and define $k_{\ell}$ by $(4.38)$ for $\ell \in\{2,3\}$. This gives $k_{1}>k_{2} \approx 12.925>k_{3} \approx 7.927>k_{4}$. According to computational scheme, this leads to $s_{2} \approx-3.407, s_{3} \approx-2.814$, and $Y_{1}>Y_{2} \approx 8.834>Y_{3} \approx 8.760>Y_{4}$. So, $j=4, \kappa(1)=4$, and $\sigma_{1}=s_{4}$, justifying the retainment of $S_{4, \ell}$ for $1 \leq \ell \leq 4$. By design, $\left(B_{4}-B_{1}\right) /\left(c_{4}-c_{1}\right)=\left(B_{4}-B_{2}\right) /\left(c_{4}-c_{2}\right)=$ $\left(B_{4}-B_{3}\right) /\left(c_{4}-c_{3}\right)$. Hence, $\kappa(2)=1$ and $\sigma_{2}=\left(B_{4}-B_{\ell}\right) /\left(c_{4}-c_{\ell}\right) \approx-4.739$ for $\ell \in\{1,2,3\}$, with the consequence that $T_{2} \approx 5.916$. There is a unique generalized $(s, S)$ policy, with $N=2, \boldsymbol{S}_{1}=S_{4,4}$, and $\boldsymbol{S}_{2}=S_{4,1}$. Moreover, it has the property that from the inventory level $\sigma_{2}$ one can order to the level $S_{4, \ell}$ from supplier $\ell$ for every $\ell \in\{1,2,3,4\}$,

Beside the last-mentioned property, the optimal policy of Example 4.27 comprises not intervening if the inventory level $x>\sigma_{1}$, ordering from supplier 4 if $\sigma_{2}<x \leq \sigma_{1}$, and ordering from supplier 1 if $x<\sigma_{2}$. Remarkably, this means that from the inventory level $\sigma_{2}$ an order can be placed with two suppliers that are otherwise excluded from the policy.

When future costs are discounted, the problem of minimizing the long-term average cost per unit time has been studied in $[15,16]$.

Remark 4.28. Given that there is a unique optimal policy minimizing the long-term average cost per unit time for the inventory model discounting future costs, and that this policy is an $(s, S)$ policy, consisting of ordering to the inventory level $S^{*}$ from the level $s^{*}$ using the single supplier $j^{*} \in \mathcal{J}$; under the assumption that for all sufficiently small $\alpha \geq 0$ the functions $\left\{f_{\ell}: \ell \in \mathcal{J}\right\}$ satisfy Hypothesis 3.3 with the same number $\gamma$ : the numbers $j, s_{j}$, and $S_{j}$ given by Theorem 4.13 are such that $j \rightarrow j^{*}, s_{j} \rightarrow s^{*}$, and $S_{j} \rightarrow S^{*}$ as $\alpha \rightarrow 0$.

The above does not exclude the possibility that the solution of the QVI (2.9) corresponds to a generalized $(s, S)$ policy that is not an $(s, S)$ policy, nor that the QVI has no solution, for arbitrarily small $\alpha>0$.

\section{Conclusion}

This paper considers an inventory control problem for a continuous-time continuous-state single-item inventory model with a general stationary demand-rate function. An inventory manager has a finite number of suppliers from which to order the item according to an impulse control policy. The control problem is formulated as a QVI. The solution of the QVI has been characterized and shown to be unique when it exists. This gives rise to an $(s, S)$ policy, a generalized $(s, S)$ policy that is not an $(s, S)$ policy, or there being no admissible optimal policy. Necessary and sufficient conditions for each case have been found.

The technical machinery adopted above could be used as a road map to tackle more elaborate models. Possibilities include the extension of models for a single supplier that lead to an $(s, S, T)$ policy to the corresponding models for several suppliers, the incorporation of the circumstance that suppliers might not being able to fulfil orders, and taking lead-times, in particular different lead-times for different suppliers, into account. Of special interest are stochastic continuous-time continuous-state inventory models. The well-known diffusion demand model is one such model.

Acknowledgements. The authors would like to thank an anonymous referee for constructive suggestions that led to the improvement of the paper.

\section{REFERENCES}

[1] K.J. Arrow, T. Harris and J. Marschak, Optimal inventory policy. Econometrica 19 (1951) 250-272.

[2] S. Benjaafar, D. Chen and Y. Yu, Optimal policies for inventory systems with concave ordering costs. Nav. Res. Logist. 65 (2018) 291-302.

[3] L. Benkherouf, On the optimality of $(s, S)$ inventory policies: a quasivariational approach. J. Appl. Math. Stoch. Anal. 158193 (2008) 1-9. 
[4] L. Benkherouf and A. Bensoussan, Optimality of an $(s, S)$ policy with compound Poisson and diffusion demands: a quasivariational inequalities approach. SIAM J. Control Optim. 48 (2009) 756-762.

[5] A. Bensoussan, Dynamic Programming and Inventory Control. IOS Press, Amsterdam (2011).

[6] A. Bensoussan and J.-L. Lions, Impulse Control and Quasi-Variational Inequalities. Gauthier-Villars, Paris (1984).

[7] A. Bensoussan, R.H. Liu and S.P. Sethi, Optimality of an $(s, S)$ policy with compound Poisson and diffusion demands: a quasi-variational inequalities approach. SIAM J. Control Optim. 44 (2005) 1650-1676.

[8] A. Bensoussan and C.S. Tapiero, Impulsive control in management: prospects and applications. J. Optim. Theory App. 37 (1982) 419-442.

[9] E.J. Fox, R. Metters and J. Semple, Optimal inventory policies with two suppliers. Oper. Res. 54 (2006) 389-393.

[10] S.K. Goyal and B.C. Giri, Recent trends in modeling of deteriorating inventory. Eur. J. Oper. Res. 134 (2001) 1-16.

[11] G. Hadley and T.M. Whitin, Analysis of Inventory Systems. Prentice-Hall, Englewood Cliffs, NJ (1963).

[12] S. He, D. Yao and H. Zhang, Optimal ordering policy for inventory systems with quantity-dependent setup costs. Math. Oper. Res. 42 (2017) 979-1006.

[13] D.L. Iglehart, Optimality of $(s, S)$ policies in the infinite horizon dynamic inventory problem. Manage. Sci. 9 (1963) $259-267$.

[14] A.G. Lagodimos, I.T. Christou and K. Skouri, Computing globally optimal $(s, S, T)$ inventory policies. Omega 40 (2012) 660-671.

[15] S. Perera, G. Janakiraman and S.-C. Niu, Optimality of $(s, S)$ policies in EOQ models with general cost structures. Int. J. Prod. Econ. 187 (2017) 216-228.

[16] S. Perera, G. Janakiraman and S.-C. Niu, Optimality of $(s, S)$ inventory policies under renewal demand and general cost structures. Prod. Oper. Manage. 27 (2018) 368-383.

[17] E.L. Porteus, On the optimality of generalized $(s, S)$ policies. Manage. Sci. 17 (1971) 411-426.

[18] E.L. Porteus, The optimality of generalized $(s, S)$ policies under uniform demand densities. Manage. Sci. 18 (1972) 644-646.

[19] E.L. Porteus, Foundations of Stochastic Inventory Theory. Stanford University Press, Stanford, CA (2002).

[20] P.A. Samuelson, A note on measurement of utility. Rev. Econ. Stud. 4 (1937) 155-161.

[21] H. Scarf, The optimality of $(s, S)$ policies in the dynamic inventory problem, edited by K.J. Arrow, S. Karlin and P. Suppes. In: Mathematical Methods in the Social Sciences 1959. Stanford University Press, Stanford, CA (1960) 196-202.

[22] A. Sulem, A solvable one-dimensional model of a diffusion inventory system. Math. Oper. Res. 11 (1986) $125-133$.

[23] A. Sulem, Explicit solution of a two-dimensional deterministic inventory problem. Math. Oper. Res. 11 (1986) $134-146$.

[24] T.L. Urban, Inventory models with inventory-level-dependent demand: a comprehensive review and unifying theory. Eur. J. Oper. Res. 162 (2005) 792-804.

[25] A.F. Veinott, On the optimality of $(s, S)$ inventory policies: new conditions and a new proof. SIAM J. Appl. Math. 14 (1966) $1067-1083$.

[26] D. Yao, X. Chao and J. Wu, Optimal control policy for a Brownian inventory system with concave ordering cost. J. Appl. Probab. 52 (2015) 909-925.

[27] D. Yao, X. Chao and J. Wu, Optimal policies for Brownian inventory systems with a piecewise linear ordering cost. IEEE Trans. Autom. Control 62 (2017) 3235-3248.

[28] E. Zabel, A note on the optimality of $(S, s)$ policies in inventory theory. Manage. Sci. 9 (1962) 123-125. 